\title{
Soret and Dufour Effects in MHD Peristalsis of Pseudoplasticnano Fluid with Porous Medium in Tapered Channel
}

\author{
Mohammed R. Salman', Ahmed M. Abdulhadi \\ University of Baghdad-College of Science-Department of Math-Baghdad-Iraq \\ Email address: mawb1967[at]gmail.com (M. R. Salman), ahm6161[at]yahoo.com (A. M. Abdulhadi)
}

\begin{abstract}
This paper is devoted to the study on peristaltic flow of a pseudoplastic fluid model in the category of non-Newtonian viscoelastic fluids through porous medium under combined effects of magnetohydrodynamic (MHD) and properties walls in a tapered asymmetric channel. Mathematical modeling comprising the momentum, pressure and energy equations has been presented and simplified through long wavelength and Reynolds number concepts. In the present study the resulting non-linear system has been computed for series solutions of stream function, velocity, temperature. Pumping characteristics, pressure gradient distribution, heat transfer coefficient, velocity, temperature distribution in the present study and trapping are further examined. In flounces of various emerging parameters have been studied graphically. It is found that Impacts of variables reflecting the salient features of wall properties on her and analyzed a trapping phenomenon is also.
\end{abstract}

Keywords: peristalsis, pseudoplastic, porous medium, Reynolds number, Hartmann number

\section{Introduction}

The peristalsis has become a topic of great in the study for biomedical and industrial applications and physiological. The influence is found in the urine passage from kidney to bladder, food moving through esophagus to stomach, blood circulation in small blood vesselsl. Latham [18], many investigations [2, 3, 19]. As well no doubt the pseudoplastic fluid falls in the category of non-Newtonian fluids. Pseudoplastic fluid is a fluid whose exhibits viscosity decreases with an increase in shear stress. They are often called shear-thinning fluids. Common examples of pseudoplastic fluids are ketchup, nail polish, molasses, whipped cream, latex paint, some silicone, oils, blood, some silicone coatings, etc. The peristaltic flow of pseudoplastic fluid in a curved channel under the influence of radial magnetic field has been inquiry by Hayat et al. [14].Recently, Hayat et al.[6] Further the peristalsis subject to magnetic field effects are significant in magnetotherapy, hyperthermia, arterial flow, cancer therapy, etc. The controlled application of low intensity and frequency pulsating fields modify the cell and tissue. Magnetic susceptible of chime is also satisfied from the heat generated by magnetic field or the ions contained in the chyme. The magnets could heat inflammations, ulceration and several diseases of intestine and uterus.

The peristaltic flow of nanofluid through a porous medium with mixed convection is analyzed by Nowar [9]. Influence of convective conditions on peristaltic transport of nonNewtonian fluids under different flow situations are discussed in refs. [8, 10]. Ellahi et al. [13] studied the peristalsis in non-uniform rectangular duct with heat and mass transfer effects. Hayat et al. [15, 17] discussed the peristaltic flow in a vertical channel filled with nanofluid by considering Soret and Dufour effects. Peristaltic transport of fourth grade fluid with convective conditions is numerically discussed by Mustafa et al. [11]. An effect of variable viscosity on peristalsis in the presence of magnetic field is studied by Abbasi et al. [6].Kothandapani and Prakash [2] studied the combined effects of radiation and magnetic field on peristaltic transport of nanofluid. The studies related to peristalsis with heat and mass transfer for Newtonian fluid have been structured in ref. [7]. Shaban and Abou-Zeid [1] investigated the peristalsis between two coaxial cylinders when inner tube is rigid and outer flexible. Heat transfer is also considered in the study.

For all such aspects in mind the purpose of present opinions and information is to discuss the magnetic field effects in peristaltic flow of pseudoplasticnano-fluid in asymmetric channel. The channel walls have compliant properties. It observed the behavior of pseudoplastic materials whose apparent viscosity or consistency decreases instantaneously with an increase in shear rate [4]. Also been noted that a study the general case of asymmetric wall oscillation in nonuniform channel may also exist in biological conduits, for example the uterus [5].

In the present work, an attempt to study the fluid flow by an asymmetric channel a particulate suspension induced by the sinusoidal peristaltic waves is made. The purpose of the present paper is to discuss the peristaltic flow of a pseudoplasticnano-fluid in the tapered asymmetric channel. The governing equations of motion, energy of nanoparticles for nano-fluids have been carried out under the assumption of long wavelength and low Reynolds number. The reduced equations are then solved analytically has also been performed for the average rise in pressure. The effect of physical parameters such as non-uniform parameter, phase difference, Hartmann number, thermal radiation parameter, thermophoresis parameter, Prandtl number are shown and discussed in detail with the help of graphs.

\section{Mathematical Formulation}

Consider the flow of an incompressible magnetoydrodynamic (MHD) pseudoplasticnano fluid in a two- 


\section{International Journal of Science and Research (IJSR) \\ ISSN (Online): 2319-7064 \\ Index Copernicus Value (2016): 79.57 | Impact Factor (2015): 6.391}

dimensional tapered asymmetric channel of width $2 \mathrm{~d}$ through a porous space. The asymmetry in channel is due to propagating waves with different amplitudes and phases. Wave speed of peristaltic waves is denoted by c. Here $\widetilde{X}-$ axis is taken along the length of channel and $\widetilde{Y}-$ axis transverse to it (see Figure (1)). A uniform magnetic field $\mathrm{B}=\left(0, \mathrm{~B}_{0}, 0\right)$ is applied. The induced magnetic field is neglected by assuming a very small magnetic Reynolds number. Also the electric field is taken absent. Heat and mass transfer is examined through convective conditions. The flow is generated by sinusoidal waves propagating along the compliant walls of channel. Let $\widetilde{Y}=\widetilde{\mathrm{H}}_{1}$ and $\widetilde{\mathrm{Y}}=\widetilde{\mathrm{H}}_{2}$ be the two walls. The geometry of the wall surfaces is given as follows:

$$
\begin{aligned}
& \widetilde{\mathrm{Y}}=\widetilde{\mathrm{H}}_{1}(\widetilde{\mathrm{X}}, \hat{\mathrm{t}}) \\
& =-d-m^{\prime} \widetilde{X}-b_{1} \sin \left[\frac{2 \pi}{\lambda}(\widetilde{X}-c t)+\phi\right], \\
& \widetilde{\mathrm{Y}}=\widetilde{\mathrm{H}}_{2}(\widetilde{\mathrm{X}}, \hat{\mathrm{t}}) \\
& =d+m^{\prime} \widetilde{X}+b_{2} \sin \left[\frac{2 \pi}{\lambda}(\widetilde{X}-c t)\right] \text {. }
\end{aligned}
$$

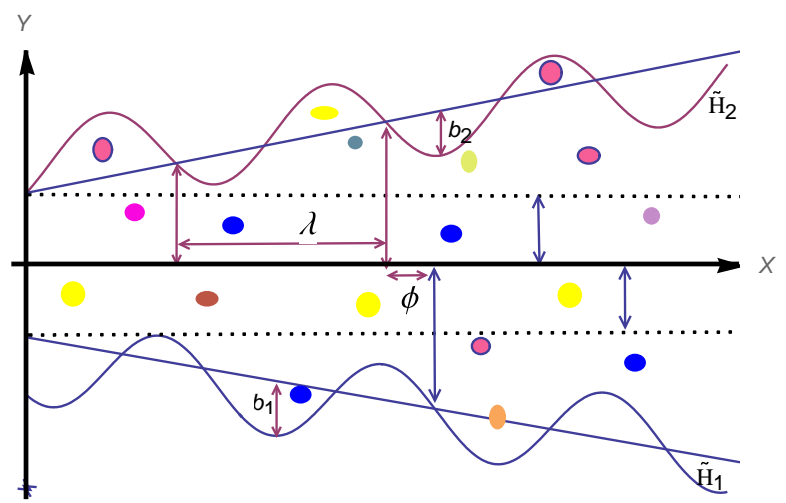

Figure 1: Geometry of the tapered asymmetric channel.

Where $d$ is the half-width of the channel, $b_{1} a_{2} b_{2}$ are the amplitudes of lower and upper walls respectively, $\mathrm{c}$ is the phase speed of the wave, $\mathrm{m}(\ll 1)$ is the non-uniform parameter, $\lambda$ is the wave length, the phase difference $\phi$ varies in the range $0 \leq \phi \leq \pi, \phi=0$ corresponds to symmetric channel with waves out of phase i.e. both walls move towards outward or inward simultaneously and further $b_{1}, b_{2}, d$ and $\phi$ satisfy the following condition at the inlet of divergent channel

$$
b_{1}^{2}+b_{2}^{2}+2 b_{1} b_{2} \cos (\phi) \leq(2 d)^{2}
$$

Here we assume the fluid to be electrically conducting in the presence of a uniform inclined magnetic field $B=\left(0, B_{0}, 0\right)$. To calculate the Lorentz force we will apply a magnetic field just in $\widetilde{Y}$ - direction and then we study the effect of it on the fluid flow as the extra stress tensor for pseudoplastic fluid [19] is given as follows:

$$
\begin{aligned}
& \tilde{\mathbf{S}}+\lambda_{1} \frac{\mathrm{D} \tilde{\mathbf{S}}}{\mathrm{D}^{\prime} \mathrm{t}} \\
& +\frac{1}{2}\left(\lambda_{1}-\mu_{1}\right)\left(A_{1} \tilde{\mathbf{S}}+\tilde{\mathbf{S}} A_{1}\right)=\mu A_{1}
\end{aligned}
$$

In which $\lambda_{1}$ and $\mu_{1}$ are the relaxation times. Also $A_{1}=$ $\left[\nabla \vec{V}+(\nabla \vec{V})^{T}\right], A_{1}$ is the first Rivlin-Ericksen tensor with the velocity gradient, and

$\mathrm{d} \tilde{\mathbf{S}} / \mathrm{d} \mathfrak{t}=\partial \tilde{\mathbf{S}} / \partial \dot{\mathrm{t}}+\overrightarrow{\mathbf{V}} \cdot \nabla \tilde{\mathbf{S}}$ and

$\mathrm{D} \tilde{\mathbf{S}} / \mathrm{D} \hat{\mathrm{t}}=\mathrm{d} \tilde{\mathbf{S}} / \mathrm{d} \hat{\mathrm{t}}-(\nabla \overrightarrow{\mathrm{V}}) \tilde{\mathbf{S}}-\tilde{\mathbf{S}}(\nabla \overrightarrow{\mathrm{V}})^{\mathrm{T}}$

Then

$\frac{\mathrm{D} \tilde{\mathbf{S}}}{\mathrm{D} \hat{\mathrm{t}}}=\partial \tilde{\mathbf{S}} / \partial \mathrm{t}+\overrightarrow{\mathbf{V}} \cdot \nabla \tilde{\mathbf{S}}-(\nabla \overrightarrow{\mathrm{V}}) \tilde{\mathbf{S}}-\tilde{\mathbf{S}}(\nabla \overrightarrow{\mathrm{V}})^{\mathrm{T}}$

From the previous matrices and the relation (4), the strees components $\widetilde{S}_{\widetilde{X} \widetilde{X}}, \widetilde{S}_{\widetilde{X} \widetilde{Y}}$ and $\widetilde{S}_{\widetilde{Y} \widetilde{Y}}$ can be obtained through the following relation:

$$
\begin{aligned}
& \widetilde{S}_{\widetilde{X} \widetilde{X}}+\lambda_{1}\left[\left(\frac{\partial}{\partial t}+\widetilde{U} \frac{\partial}{\partial \widetilde{X}}+\widetilde{V} \frac{\partial}{\partial \widetilde{Y}}\right) \widetilde{S}_{\widetilde{X} \widetilde{X}}-2 \widetilde{S}_{\widetilde{X} \widetilde{X}} \frac{\partial \widetilde{U}}{\partial \widetilde{X}}-\right. \\
& 2 S X Y \partial U \partial Y+12 \lambda 1-\mu 14 S X X U \partial X+2 S X Y \partial \partial Y+\partial V \partial X= \\
& 2 \mu \frac{\partial \widetilde{U}}{\partial \widetilde{X}} \\
& \widetilde{S}_{\widetilde{X} \widetilde{Y}}+\lambda_{1}\left[\left(\frac{\partial}{\partial \tilde{t}}+\widetilde{U} \frac{\partial}{\partial \widetilde{X}}+\widetilde{V} \frac{\partial}{\partial \widetilde{Y}}\right) \tilde{S}_{\widetilde{X} \widetilde{Y}}-\widetilde{S}_{\widetilde{X} \widetilde{X}} \frac{\partial \widetilde{V}}{\partial \widetilde{X}}-\widetilde{S}_{\widetilde{Y} \widetilde{Y}} \frac{\partial \widetilde{U}}{\partial \widetilde{Y}}\right]+ \\
& \frac{1}{2}\left(\lambda_{1}-\mu_{1}\right)\left(\widetilde{S}_{\widetilde{X} \widetilde{X}}+\widetilde{S}_{\widetilde{Y} \widetilde{Y}}\right)\left(\frac{\partial \widetilde{U}}{\partial \widetilde{Y}}+\frac{\partial \widetilde{V}}{\partial \widetilde{X}}\right)=\mu\left(\frac{\partial \widetilde{U}}{\partial \widetilde{Y}}+\frac{\partial \widetilde{V}}{\partial \widetilde{X}}\right) \\
& \widetilde{S}_{\widetilde{Y} \widetilde{Y}}+\lambda_{1}\left[\left(\frac{\partial}{\partial t}+\widetilde{U} \frac{\partial}{\partial \widetilde{X}}+\widetilde{V} \frac{\partial}{\partial \widetilde{Y}}\right) \widetilde{S}_{\widetilde{Y} \widetilde{Y}}-2 \widetilde{S}_{\widetilde{Y} \widetilde{X}} \frac{\partial \widetilde{V}}{\partial \widetilde{X}}-\right. \\
& 2 S Y Y \partial V \partial Y+12 \lambda 1-\mu 12 S X Y \partial U Y+\partial V \partial X+4 S Y Y \partial V \partial Y= \\
& 2 \mu \frac{\partial \widetilde{V}}{\partial \widetilde{Y}}
\end{aligned}
$$

\section{Constitutive Equations}

The basic equations governing the Non-Newtonian incompressible (MHD) pseudoplasticnano-fluid in 2dimensional flow.

The continuity equation is given by

$$
\frac{\partial \widetilde{U}}{\partial \tilde{X}}+\frac{\partial \widetilde{V}}{\partial \tilde{Y}}=0
$$

The momentum equations are:

In the $\tilde{X}-$ direction

$$
\begin{gathered}
\rho_{f}\left[\frac{\partial \widetilde{U}}{\partial \dot{t}}+\widetilde{U} \frac{\partial \widetilde{U}}{\partial \tilde{X}}+\tilde{V} \frac{\partial \widetilde{U}}{\partial \tilde{Y}}\right]=-\frac{\partial \tilde{P}}{\partial \tilde{X}}+\frac{\partial}{\partial \tilde{X}}\left(\tilde{S}_{\tilde{X} \tilde{X}}\right) \\
+\frac{\partial}{\partial \tilde{Y}}\left(\tilde{S}_{\tilde{X} Y}\right)-\boldsymbol{\sigma} B_{0}^{2} \widetilde{U}-\frac{\mu}{K_{0}} \widetilde{U},
\end{gathered}
$$

In the $\tilde{Y}-$ direction

$$
\begin{aligned}
& \rho_{f}\left[\frac{\partial \tilde{V}}{\partial \dot{t}}+\widetilde{U} \frac{\partial \tilde{V}}{\partial \tilde{X}}+\tilde{V} \frac{\partial \tilde{V}}{\partial \tilde{Y}}\right]=-\frac{\partial \tilde{P}}{\partial \tilde{Y}}+\frac{\partial}{\partial \tilde{X}}\left(\tilde{S}_{\tilde{Y} \tilde{X}}\right) \\
& +\frac{\partial}{\partial \tilde{Y}}\left(\tilde{S}_{\tilde{Y} \tilde{Y}}\right)-\frac{\mu}{K_{0}} \tilde{V} .
\end{aligned}
$$

The nano-particle temperature equation is determined by

$$
\begin{aligned}
& (\rho \dot{c})_{f}\left[\frac{\partial \tilde{T}}{\partial \tilde{t}}+\widetilde{U} \frac{\partial \tilde{T}}{\partial \tilde{X}}+\tilde{V} \frac{\partial \tilde{T}}{\partial \tilde{Y}}\right]= \\
& k\left[\frac{\partial^{2} \tilde{T}}{\partial \tilde{X}^{2}}+\frac{\partial^{2} \tilde{T}}{\partial \tilde{Y}^{2}}\right]+(\rho \dot{c})_{p} \frac{D_{T}}{T_{m}}\left[\left(\frac{\partial \tilde{T}}{\partial \tilde{X}}\right)^{2}+\left(\frac{\partial \tilde{T}}{\partial \tilde{Y}}\right)^{2}\right]
\end{aligned}
$$




\section{International Journal of Science and Research (IJSR) \\ ISSN (Online): 2319-7064}

Index Copernicus Value (2016): 79.57 | Impact Factor (2015): 6.391

In above equations $t$ is the dimensional time, $\tilde{S}_{\tilde{X} \tilde{X}}, \tilde{S}_{\tilde{X} \tilde{Y}}, \tilde{S}_{\tilde{Y} \tilde{X}}, \tilde{S}_{\tilde{Y} \tilde{Y}}$ the components of stress tensor, $\boldsymbol{\sigma}$ the fluid electrical conductivity, $\widetilde{U}$ and $\tilde{V}$ are the velocity components in the axial and transverse directions respectively, $k$ the thermal conductivity of fluid, $B_{0}$ is the magnetic parameter, $\boldsymbol{\rho}_{\boldsymbol{f}}$ the density of fluid, $\boldsymbol{\rho}_{\boldsymbol{p}}$ the density of nano-particles, $\tilde{T}$ the temperature of fluid, $T_{m}$ the fluid mean temperature, $D_{T}$ the themophoretic diffusion coefficient, $K_{0}$ the permeability parameter.

Now, for $\widetilde{U}$ and $\tilde{V}$ be the respective velocity components along $\tilde{X}$ and $\tilde{Y}$ directions in the fixed fram, respectively. For the unsteady two-dimensional flow, the velocity components may be written $\widetilde{V}=(\widetilde{U}(\tilde{X}, \tilde{Y}), \tilde{V}(\tilde{X}, \tilde{Y}), 0)$.

The temperature function may be written $T=T(\tilde{X}, \tilde{Y})$.

The appropriate boundary conditions comprising wall no-slip and convective boundary conditions are given as follows:

$$
\begin{aligned}
& U=0, \tilde{T}=T_{0} \text { at } \tilde{Y}=\widetilde{H}_{1}, \\
& U=0, \tilde{T}=T_{0} \text { at } \tilde{Y}=\widetilde{H}_{1} .
\end{aligned}
$$

Introducing the wave frame having coordinates $(\tilde{x}, \tilde{y})$ which travel in the $\tilde{x}$-direction with the same wave velocity $c$, and the unsteady flow in the laboratory frame $(\tilde{X}, \tilde{Y})$ can be treated as steady. The coordinates and velocities in the two frames are related by

$$
\begin{aligned}
& \tilde{x}=\tilde{X}-c \hat{t}, \tilde{y}=\tilde{Y}, \tilde{u}(\tilde{x}, \tilde{y})=\widetilde{U}(\tilde{X}, \tilde{Y}, \hat{t})-c, \\
& \tilde{v}(\tilde{x}, \tilde{y})=\tilde{V}(\tilde{X}, \tilde{Y}, \hat{t}) .(14)
\end{aligned}
$$

Where $\tilde{u}, \tilde{v}$ are the velocity components in the wave frame $(\tilde{x}, \tilde{y})$.

Using the transformations in Eq.(14), into constitutive relations Eqs.(5)-(7) and governing Eqs.(8)-(11) we have the following form.

$$
\begin{aligned}
& \frac{\partial(\tilde{u}+c)}{\partial(\tilde{x}+c \tilde{t})}+\frac{\partial \tilde{v}}{\partial \tilde{y}}=0 \\
& \rho_{f}\left[\frac{\partial(\tilde{u}+c)}{\partial \tilde{t}}+(\tilde{u}+c) \frac{\partial(\tilde{u}+c)}{\partial(\tilde{x}+c \tilde{t})}+\tilde{v} \frac{\partial(\tilde{u}+c)}{\partial \tilde{y}}\right]= \\
& -\frac{\partial p}{\partial(\tilde{x}+c \hat{t})}+\frac{\partial}{\partial(\tilde{x}+c \hat{t})}\left(\tilde{S}_{\tilde{x} \tilde{x}}\right)+\frac{\partial}{\partial \tilde{y}}\left(\tilde{S}_{\tilde{x} \tilde{y}}\right)- \\
& \dot{\boldsymbol{\sigma}} B_{\mathbf{0}}^{2}(\tilde{u}+c)-\frac{\mu}{K_{0}}(\tilde{u}+c) \\
& \rho_{f}\left[\frac{\partial \tilde{v}}{\partial \tilde{t}}+(\tilde{u}+c) \frac{\partial \tilde{v}}{\partial(\tilde{x}+c \tilde{t})}+\tilde{v} \frac{\partial \tilde{v}}{\partial \tilde{y}}\right]=-\frac{\partial p}{\partial \tilde{y}}+ \\
& \frac{\partial}{\partial(\tilde{x}+c \tilde{t})}\left(\tilde{S}_{\tilde{x} \tilde{y}}\right)+\frac{\partial}{\partial \tilde{y}}\left(\tilde{S}_{\tilde{y} \tilde{y}}\right)-\frac{\mu}{K_{0}} \tilde{v} \\
& (\rho)_{f}\left[\frac{\partial \tilde{T}}{\partial \dot{t}}+(\tilde{u}+c) \frac{\partial \tilde{T}}{\partial(\tilde{x}+c \hat{t})}+\tilde{v} \frac{\partial \tilde{T}}{\partial \tilde{y}}\right] \\
& =k\left[\frac{\partial^{2} \tilde{T}}{\partial(\tilde{x}+c t)^{2}}+\frac{\partial^{2} \tilde{T}}{\partial \tilde{y}^{2}}\right]+(\rho \dot{c})_{p} \frac{D_{T}}{T_{m}}\left[\left(\frac{\partial \tilde{T}}{\partial(\tilde{x}+c \hat{t})}\right)^{2}+\right. \\
& \partial T \partial y 2
\end{aligned}
$$

The extra stress components $\tilde{S}_{\tilde{X} \tilde{X}}, \tilde{S}_{\tilde{X} \tilde{Y}}$ and $\tilde{S}_{\tilde{Y} \tilde{Y}}$ satisfy the following relations:
$\tilde{S}_{\tilde{x} \tilde{x}}+\lambda_{1}\left[\left(\frac{\partial}{\partial \tilde{t}}+(\tilde{u}+c) \frac{\partial}{\partial(\tilde{x}+c t)}+\tilde{v} \frac{\partial}{\partial \tilde{y}}\right) \tilde{S}_{\tilde{x} \tilde{x}}\right.$

$2 S x x \partial u+c \partial x+c t-2 S x y \partial u+c \partial y+12 \lambda 1-\mu 14 S x x \partial u+c \partial x$ $+c t+2 S x y \partial u+c \partial y+\partial v \partial x+c t=2 \mu \partial u+c \partial x+c t$

(19)

$\tilde{S}_{\tilde{x} \tilde{y}}+\lambda_{1}\left[\left(\frac{\partial}{\partial \tilde{t}}+(\tilde{u}+c) \frac{\partial}{\partial(\tilde{x}+c \tilde{t})}+\tilde{v} \frac{\partial}{\partial \tilde{y}}\right) \tilde{S}_{\tilde{x} \tilde{y}}-\right.$ $S x x \partial v \partial x+c t-S y y \partial u+c \partial y+12 \lambda 1-\mu 1 S x x+S y y \partial u+c \partial$ $y+\partial v \partial x+c t=\mu \partial u+c \partial y+\partial v \partial x+c t$

$\tilde{S}_{\tilde{y} \tilde{y}}+\lambda_{1}\left[\left(\frac{\partial}{\partial \tilde{t}}+(\tilde{u}+c) \frac{\partial}{\partial(\tilde{x}+c t)}+\tilde{v} \frac{\partial}{\partial \tilde{y}}\right) \tilde{S}_{\tilde{y} \tilde{y}}-\right.$ $2 S y x \partial v \partial x+c t-2 S y y \partial v \partial y+12 \lambda 1-\mu 12 S x y \partial u+c \partial y+\partial$ $v \partial x+c t+4 S y y \partial v \partial y=2 \mu \partial v \partial y$

In order to describe the fluid flow in a non-dimensional form, we introduce the following quantities in Eqs.(15)-(21):

$$
\begin{gathered}
x=\frac{\tilde{x}}{\lambda}, y=\frac{\tilde{y}}{d}, t=\frac{c \dot{t}}{\lambda}, u=\frac{\tilde{u}}{c}, v=\frac{\tilde{v}}{c}, \\
\delta=\frac{d}{\lambda}, h_{1}=\frac{\widetilde{H}_{1}}{d}, h=\frac{\widetilde{H}_{2}}{d}, \theta=\frac{T-T_{0}}{T_{1}-T_{0}} \\
p=\frac{d^{2} \tilde{P}}{\lambda \mu c}, S_{i j}=\frac{d}{c \mu} \tilde{S}_{\tilde{i} \tilde{j}}, \lambda_{1}^{*}=\frac{\lambda_{1} c}{d}, \mu_{1}^{*}=\frac{\mu_{1} c}{d}, \\
K=\frac{K_{0}}{d^{2}}, R_{r}=\frac{\rho_{f} c d}{\mu}, a=\frac{b_{1}}{d}, b=\frac{b_{2}}{d}, \\
m=\frac{\dot{m} \lambda}{d}, P_{r}=\frac{\mu \dot{c}_{f}}{k}, N_{t}=\frac{\dot{\tau} D_{T}\left(T_{1}-T_{0}\right)}{T_{m} v}, \\
M=\sqrt{\frac{\dot{\sigma}}{\mu}} d B_{0} .
\end{gathered}
$$

Where $\psi$ is the stream function, $x$ is the non-dimensional axial coordinate, $y$ is the non-dimensional transverse coordinate, $t$ is the dimensionless time, $u$ and $v$ are nondimensional axial and transverse velocity components respectively, $p$ is the dimensionless pressure, $a$ and $b$ are amplitudes of upper and lower walls, $\delta$ is the wave number, $m$ is the non-uniform parameter, $K$ is the Darcy number, $R_{e}$ is the Reynolds number, $v$ is the nanofluid kinematic viscosity, $\dot{\tau}=(\rho \dot{c})_{p} /(\rho c)_{f}$ is the ratio of the effective heat capacity of nanoparticle material and heat capacity of the fluid, $\theta$ is the dimensionless temperature, $P_{r}$ is the Prandtl number, $M$ is the Hartmann number, $\boldsymbol{\rho}_{p}$ the density of nanoparticles, $N_{t}$ is the thermophoresis parameter.

And use the stream function ( $u=\frac{\partial \psi}{\partial y}, v=-\delta \frac{\partial \psi}{\partial x}$ ) we obtain the dimensionless continuity and motion equations which are

$$
\begin{aligned}
& R_{e} \delta\left[\frac{\partial}{\partial t} \frac{\partial \psi}{\partial y}+\left(\frac{\partial \psi}{\partial y}+1\right) \frac{\partial^{2} \psi}{\partial x \partial y}-\frac{\partial \psi}{\partial x} \frac{\partial^{2} \psi}{\partial x \partial y}\right]=-\frac{\partial p}{\partial x}+ \\
& \delta \frac{\partial}{\partial x}\left(S_{x x}\right)+\frac{\partial}{\partial y}\left(S_{x y}\right)-\left(M^{2}+\frac{1}{K}\right)\left(\frac{\partial \psi}{\partial y}+1\right) \\
& -R_{e} \delta^{3}\left[\left(\frac{\partial \psi}{\partial y}+1\right) \frac{\partial^{2} \psi}{\partial x^{2}}+\frac{\partial \psi}{\partial x} \frac{\partial^{2} \psi}{\partial x \partial y}\right]=-\frac{\partial p}{\partial y}+\delta^{2} \frac{\partial}{\partial x}\left(S_{x y}\right)+ \\
& \delta \frac{\partial}{\partial y}\left(S_{y y}\right)+\frac{\delta^{2}}{K} \frac{\partial \psi}{\partial x}
\end{aligned}
$$




$$
\begin{aligned}
& R_{e} \delta\left[\frac{\partial \theta}{\partial t}+\left(\frac{\partial \psi}{\partial y}+1\right) \frac{\partial \theta}{\partial x}-\frac{\partial \psi}{\partial x} \frac{\partial \theta}{\partial y}\right]=\frac{1}{P_{r}}\left[\delta^{2} \frac{\partial^{2} \theta}{\partial x^{2}}+\frac{\partial^{2} \theta}{\partial y^{2}}\right]+ \\
& N_{t}\left[\delta^{2}\left(\frac{\partial \theta}{\partial x}\right)^{2}+\left(\frac{\partial \theta}{\partial y}\right)^{2}\right]
\end{aligned}
$$

With non-dimensional stress components are:

$S_{x x}+\lambda_{1}\left\{\delta\left[\frac{\partial}{\partial t}+\left(\frac{\partial \psi}{\partial y}+1\right) \frac{\partial}{\partial x}-\frac{\partial \psi}{\partial x} \frac{\partial}{\partial y}\right] S_{x x}-\right.$

$2 \delta S x x \partial 2 \psi \partial x \partial y-2 S x y \partial 2 \psi \partial y 2+12 \lambda 1-\mu 14 \delta S x x \partial 2 \psi$ $\partial x \partial y+2 S x y \partial 2 \psi \partial y 2-\delta 2 \partial 2 \psi \partial x 2=2 \delta \partial 2 \psi \partial x \partial y$ (26)

$S_{x y}+\lambda_{1}\left\{\delta\left[\frac{\partial}{\partial t}+\left(\frac{\partial \psi}{\partial y}+1\right) \frac{\partial}{\partial x}-\frac{\partial \psi}{\partial x} \frac{\partial}{\partial y}\right] S_{x y}+\right.$ $\delta 2 S x x \partial 2 \psi \partial x 2-S y y \partial 2 \psi \partial y 2+12 \lambda 1-\mu 1 S x x+S y y \partial 2 \psi$ $\partial y 2-\delta 2 \partial 2 \psi \partial x 2=\partial 2 \psi \partial y 2-\delta 2 \partial 2 \psi \partial x 2$ (27)

$S_{y y}+\lambda_{1}\left\{\delta\left[\frac{\partial}{\partial t}+\left(\frac{\partial \psi}{\partial y}+1\right) \frac{\partial}{\partial x}-\frac{\partial \psi}{\partial x} \frac{\partial}{\partial y}\right] S_{y y}+\right.$ $2 \delta 2 S x y \partial 2 \psi \partial x 2+2 \delta S y y \partial 2 \psi \partial x \partial y+12 \lambda 1-\mu 1-4 \delta S y y \partial 2$ $\psi \partial x \partial y+2 S x y \partial 2 \psi \partial y 2-\delta 2 \partial 2 \psi \partial x 2=-2 \delta \partial 2 \psi \partial x \partial y$ (28)

Similarly Eqs.(12) and (13) become

$$
\begin{array}{r}
\frac{\partial \psi}{\partial y}=0, \theta=0 \text { at } y=h_{1}, \\
\frac{\partial \psi}{\partial y}=0, \theta=1 \text { at } y=h_{2},
\end{array}
$$

Eqs.(23)-(28) under long wavelength and low Reynolds number approximation lead to following set of equations:

$$
\begin{aligned}
& \frac{\partial p}{\partial x}=\frac{\partial}{\partial y}\left(S_{x y}\right)-\left(M^{2}+\frac{1}{K}\right)\left(\frac{\partial \psi}{\partial y}+1\right) \\
& \frac{\partial p}{\partial y}=0 \\
& \frac{\partial^{2} \theta}{\partial y^{2}}+P_{r} N_{t}\left(\frac{\partial \theta}{\partial y}\right)^{2}=0
\end{aligned}
$$

With

$$
\begin{aligned}
& S_{x x}=\left(\lambda_{1}+\mu_{1}\right) S_{x y} \frac{\partial^{2} \psi}{\partial y^{2}} \\
& S_{x y}++\frac{1}{2}\left(\lambda_{1}-\mu_{1}\right)\left(S_{x x}+S_{y y}\right) \frac{\partial^{2} \psi}{\partial y^{2}}-\lambda_{1} S_{y y} \frac{\partial^{2} \psi}{\partial y^{2}} \\
& =\frac{\partial^{2} \psi}{\partial y^{2}} \\
& S_{y y}=-\left(\lambda_{1}-\mu_{1}\right) S_{x y} \frac{\partial^{2} \psi}{\partial y^{2}}
\end{aligned}
$$

Eq.(32) shows that $p$ is not a function of $y$. Eliminating pressure from Eqs.(31) and (32) and simplifying Eqs.(34)(36), we get the following system:

$$
\frac{\partial^{2}}{\partial y^{2}}\left(S_{x y}\right)-\left(M^{2}+\frac{1}{K}\right) \frac{\partial^{2} \psi}{\partial y^{2}}=0
$$

$$
\begin{aligned}
& \frac{\partial^{2} \theta}{\partial y^{2}}+P_{r} N_{t}\left(\frac{\partial \theta}{\partial y}\right)^{2}=0 \\
& S_{x y}=\frac{\frac{\partial^{2} \psi}{\partial y^{2}}}{1-\xi\left(\frac{\partial^{2} \psi}{\partial y^{2}}\right)^{2}}
\end{aligned}
$$

$$
\left.\begin{array}{rl}
\psi & =-\frac{F}{2}, \frac{\partial \psi}{\partial y}=0, \theta=0 \text { at } \\
y & =h_{1}=-1-m(x+t)-a \sin [2 \pi x+\phi], \\
\psi & =\frac{F}{2}, \frac{\partial \psi}{\partial y}=0, \theta=1 \text { at } \\
y & =h_{2}=1+m(x+t)+b \sin [2 \pi x] .
\end{array}\right]
$$

Here $\xi=\left(\mu_{1}^{2}-\lambda_{1}^{2}\right)$ is the pseudoplastic fluid parameter and $F$ represents the dimensionless mean flows. Here.

$+b \sin [2 \pi x](42)$

$$
F(x, t)=Q+a \sin [2 \pi x+\phi]
$$

Where $Q$ is the dimensionless mean flow in the wave frame, Here

$$
\begin{aligned}
& F=\int_{h_{1}(x)}^{h_{2}(x)} \frac{\partial \psi}{\partial y} d y=\psi\left(h_{2}\right)- \\
& \psi\left(h_{1}\right) \\
& Q \equiv \frac{\tilde{q}}{c d},(43) \\
& \tilde{q}=\int_{\widetilde{H}_{1}(\tilde{X}, \tilde{t})}^{\widetilde{H}_{2}(\tilde{X}, \tilde{t})} \tilde{u}(\tilde{x}, \tilde{y}) d \tilde{y}
\end{aligned}
$$

Here it is pointed out that the conditions on $\psi$ satisfy Eq.(43) and the conditions on $\partial \psi / \partial y$ are no-slip.

The coefficient of heat transfer at the right wall is given by

$$
Z(x)=\frac{\partial h_{1}}{\partial x}\left(\frac{\partial \theta}{\partial y}\right)_{y=h_{1}}
$$

The non-dimensional average rise in pressure $\Delta p$ per wavelength is given by

$$
\Delta p=\int_{0}^{1} \frac{\partial p}{\partial x} d x
$$

\section{Perturbed System and Perturbation Solutions}

After using binomial expansion for small pseudoplastic fluid parameter $\xi$. Eqs.(37) and (38) and let $\left(N^{2}=M^{2}+\frac{1}{K}\right)$ may be simplified to

$$
\begin{aligned}
& \frac{\partial p}{\partial x} \\
& =\frac{\partial^{3} \psi}{\partial y^{3}}+\xi \frac{\partial}{\partial y}\left[\left(\frac{\partial^{2} \psi}{\partial y^{2}}\right)^{3}\right] \\
& +\xi^{2} \frac{\partial}{\partial y}\left[\left(\frac{\partial^{2} \psi}{\partial y^{2}}\right)^{4}\right]- \\
& N^{2}\left(\frac{\partial \psi}{\partial y}+1\right)(47)
\end{aligned}
$$




$$
\begin{aligned}
& \frac{\partial^{4} \psi}{\partial y^{4}} \\
& +3 \xi\left[\left(\frac{\partial^{2} \psi}{\partial y^{2}}\right)^{2} \frac{\partial^{4} \psi}{\partial y^{4}}\right. \\
& \left.+2\left(\frac{\partial^{3} \psi}{\partial y^{3}}\right)^{2} \frac{\partial^{2} \psi}{\partial y^{2}}\right] \\
& -N^{2} \frac{\partial^{2} \psi}{\partial y^{2}}=0 .
\end{aligned}
$$

In order to solve the present problem, we expand the flow quantities in a power series of $\xi$ and $\operatorname{Pr}$ as follows:

$$
\left.\begin{array}{l}
\Omega=\Omega_{\circ}+\xi \Omega_{1}+O\left(\xi^{2}\right) \\
\Omega=\Omega_{\circ}+\operatorname{Pr} \Omega_{1}+O\left(\operatorname{Pr}^{2}\right)
\end{array}\right]
$$

Substituting Eq.(49) into Eq.(38) and Eqs.(47)-(48) and boundary conditions (40) and (41) and then equating the like powers of $\xi$ and Pr we get:

$$
\begin{aligned}
& \frac{\partial^{4} \psi_{\circ}}{\partial y^{4}}-N^{2} \frac{\partial^{2} \psi_{\circ}}{\partial y^{2}}=0, \\
& \frac{\partial p_{0}}{\partial x}=\frac{\partial^{3} \psi_{0}}{\partial y^{3}}-N^{2}\left(\frac{\partial \psi_{0}}{\partial y}+1\right)=0, \\
& \frac{\partial^{2} \theta_{0}}{\partial y^{2}}=0 .
\end{aligned}
$$

With corresponding boundary conditions

$$
\begin{aligned}
& \psi_{0}=-\frac{F_{0}}{2}, \frac{\partial \psi_{0}}{\partial y}=0, \theta_{0}=0, \text { at } y=h_{1} \text {, } \\
& \psi_{0}=\frac{F_{\circ}}{2}, \frac{\partial \psi_{0}}{\partial y}=0, \theta_{0}=1, \text { at } y=h_{2} \text {. } \\
& \frac{\partial^{4} \psi_{1}}{\partial y^{4}}+3 \frac{\partial^{4} \psi_{0}}{\partial y^{4}}\left(\frac{\partial^{2} \psi_{0}}{\partial y^{2}}\right)^{2}+ \\
& 6\left(\frac{\partial^{3} \psi_{0}}{\partial y^{3}}\right)^{2}\left(\frac{\partial^{2} \psi_{0}}{\partial y^{2}}\right)-N^{2} \frac{\partial^{2} \psi_{1}}{\partial y^{2}}=0, \\
& \frac{\partial p_{1}}{\partial x}-\frac{\partial^{3} \psi_{1}}{\partial y^{3}}-3 \frac{\partial^{3} \psi_{0}}{\partial y^{3}}\left(\frac{\partial^{2} \psi_{0}}{\partial y^{2}}\right)^{2}+ \\
& N^{2} \frac{\partial \psi_{1}}{\partial y}=0 \text {, } \\
& \frac{\partial^{2} \theta_{1}}{\partial y^{2}}+N_{t}\left(\frac{\partial \theta_{0}}{\partial y}\right)^{2}=0,
\end{aligned}
$$

With corresponding boundary conditions

$$
\begin{gathered}
\psi_{1}=-\frac{F_{1}}{2}, \frac{\partial \psi_{1}}{\partial y}=0, \theta_{1}=0 \text { at } y=h_{1}, \\
\psi_{1}=\frac{F_{1}}{2}, \frac{\partial \psi_{1}}{\partial y}=0, \theta_{1}=1 \text { at } y \\
=h_{2} .
\end{gathered}
$$

The solutions of Eqs.(50)-(52) are given by

$$
\boldsymbol{\psi}_{0}=C_{3}+C_{4} y+\frac{C_{1} e^{-y N}+C_{2} e^{y N}}{N^{2}}
$$

$$
\begin{gathered}
\boldsymbol{u}_{\mathbf{0}}=\frac{\partial \psi_{0}}{\partial y}=C_{4}+\frac{-C_{1} e^{-y N}+C_{2} e^{y N}}{N}, \\
\frac{\partial \boldsymbol{P}_{\mathbf{0}}}{\boldsymbol{\partial x}}=N\left(\begin{array}{c}
\left(-C_{1} e^{-y N}+C_{2} e^{y N}\right)- \\
\left(1+C_{4}+\frac{-C_{1} e^{-y N}+C_{2} e^{y N}}{N}\right)
\end{array}\right), \\
=\frac{-y-1-m(t+x)-a \operatorname{Sin}[2 \pi x+\phi]}{-2-2 m(t+x)-b \operatorname{Sin}[2 \pi x]-a \operatorname{Sin}[2 \pi x+\phi]}
\end{gathered}
$$

The heat transfer coefficient can be expressed in the form

$$
\begin{aligned}
& \boldsymbol{Z}_{\mathbf{0}}=\frac{\partial h_{1}}{\partial x}\left(\frac{\partial \theta_{0}}{\partial y}\right)_{y=h_{1}} \\
& =-\frac{-m-2 a \pi \cos [2 \pi x+\phi]}{-2-2 m(t+x)-b \sin [2 \pi x]-a \sin [2 \pi x+\phi]}
\end{aligned}
$$

Furthermore, the non-dimensionless average rise in $\Delta p_{0}$ per wavelength at this order is

$$
\Delta \boldsymbol{p}_{0}=\int_{0}^{1}\left(\frac{\partial p_{0}}{\partial x}\right)_{y=0} d x .
$$

Substituting the zeroth-order solution Eq.(56) into Eqs.(53), (54) and then solving the resulting system with the corresponding boundary conditions, we get

$$
\begin{aligned}
& \boldsymbol{\psi}_{\mathbf{1}}=C_{7}+y C_{8} \\
& +\frac{1}{8 N}\left(\begin{array}{c}
-2 e^{-y N}\left(\begin{array}{c}
-6 C_{1}^{2} c 2 y- \\
\frac{15 C_{1}{ }^{2} C_{2}+4 C_{5}}{N}
\end{array}\right) \\
+2 e^{y N}\left(\begin{array}{c}
-6 C_{1} C_{2}^{2} y+ \\
15 C_{1} C_{2}^{2}+4 C_{6} \\
N
\end{array}\right)- \\
\frac{C_{1}^{3} e^{-3 y N}}{N}-\frac{C_{2}{ }^{3} e^{3 y N}}{N}
\end{array}\right) \\
& \boldsymbol{u}_{\mathbf{1}}=\frac{\partial \psi_{1}}{\partial y} \\
& =C_{8}+\frac{1}{8 N}\left(\begin{array}{c}
3 C_{1}{ }^{3} e^{-3 y N} \\
+12 C_{1}{ }^{2} C_{2} e^{-y N} \\
-12 C_{1} C_{1}{ }^{2} e^{y N}-3 C_{1}{ }^{3} e^{3 y N} \\
+2 e^{-y N}\left(-6 C_{1}{ }^{2} C_{2} y\right. \\
\left.-\frac{15 C_{1}{ }^{2} C_{2}+4 C_{5}}{N}\right) N \\
+2 e^{y N}\left(-6 C_{1} C_{2}{ }^{2} y\right. \\
\left.+\frac{15 C_{1} C_{2}{ }^{2}+4 C_{6}}{N}\right) N
\end{array}\right)
\end{aligned}
$$

$$
\begin{aligned}
& \begin{array}{l}
\frac{\boldsymbol{d} \boldsymbol{p}_{\mathbf{1}}}{\boldsymbol{d} \boldsymbol{x}} \\
=\frac{3\left(C_{1} e^{-y N} N^{2}+C_{2} e^{y N} N^{2}\right)^{2}\left(-C_{1} e^{-y N} N^{3}+C_{2} e^{y N} N^{3}\right)}{N^{6}} \\
+\frac{1}{8 N}\left(27 C_{1}{ }^{3} e^{-3 y N} N^{2}+36 C_{1}{ }^{2} C_{2} e^{-y N} N^{2}\right. \\
\quad-36 C_{1} C_{2}{ }^{2} e^{y N} N^{2}-27 C_{2}{ }^{3} e^{3 y N} N^{2} \\
\quad+2 e^{-y N}\left(-6 C_{1}{ }^{2} C_{2} y-\frac{15 C_{1}{ }^{2} C_{2}+4 C_{5}}{N}\right) N^{3}
\end{array} \\
& \left.+2 e^{y N}\left(-6 C_{1} C_{2}{ }^{2} y+\frac{15 C_{1} C_{2}{ }^{2}+4 C_{6}}{N}\right) N^{3}\right) \\
& -N^{2}\left(C_{8}+\frac{1}{8 N}\left(3 C_{1}{ }^{3} e^{-3 y N}+12 C_{1}{ }^{2} C_{2} e^{-y N}\right.\right.
\end{aligned}
$$

\section{Volume 6 Issue 12, December 2017}

\section{www.ijsr.net}

Licensed Under Creative Commons Attribution CC BY 


\section{International Journal of Science and Research (IJSR) \\ ISSN (Online): 2319-7064}

Index Copernicus Value (2016): 79.57 | Impact Factor (2015): 6.391

$$
\begin{aligned}
& -12 C_{1} C_{2}{ }^{2} e^{y N}-3 C_{2}{ }^{3} e^{3 y N} \\
& +2 e^{-y N}\left(-6 C_{1}{ }^{2} C_{2} y-\frac{15 C_{1}{ }^{2} C_{2}+4 C_{5}}{N}\right) N \\
& \left.\left.+2 e^{y N}\left(-6 C_{1} C_{2}{ }^{2} y+\frac{15 C_{1} C_{2}{ }^{2}+4 C_{6}}{N}\right) N\right)\right) \\
& \boldsymbol{\theta}_{\mathbf{1}} \\
& =-\frac{y^{2} N_{t}}{2(-2-2 m(t+x)-b \operatorname{Sin}[2 \pi x]-a \operatorname{Sin}[2 \pi x+\phi} \\
& -(y(-2(1+m(t+x)+b \operatorname{Sin}[2 \pi x]) \\
& +2(-1-m(t+x)-a \operatorname{Sin}[2 \pi x+\phi]) \\
& -(1+m(t+x)+b \operatorname{Sin}[2 \pi x]) N_{t} \\
& \left.\left.-(-1-m(t+x)-a \operatorname{Sin}[2 \pi x+\phi]) N_{t}\right)\right) / \\
& (2(-b \operatorname{Sin}[2 \pi x]-a \operatorname{Sin}[2 \pi x+\phi]) \\
& -(2(1+m(t+x)+b \operatorname{Sin}[2 \pi x])(-1 \\
& -m(t+x)-a \operatorname{Sin}[2 \pi x+\phi]) \\
& -2(-1-m(t+x)-a \operatorname{Sin}[2 \pi x+\phi])^{2} \\
& +(1+m(t+x)+b \operatorname{Sin}[2 \pi x])(-1 \\
& \left.-m(t+x)-a \operatorname{Sin}[2 \pi x+\phi]) N_{t}\right) /(2 \\
& \quad(-2-2 m(t+x)-b \operatorname{Sin}[2 \pi x]-a \operatorname{Sin}[2 \pi x \\
& \left.\left.\quad+\phi]^{2}\right)\right)
\end{aligned}
$$

$\mathrm{Tt}$ The heat transfer coefficient at this order is given by

$$
\begin{aligned}
& \boldsymbol{Z}_{\mathbf{1}}=\frac{\partial h_{1}}{\partial x}\left(\frac{\partial \theta_{1}}{\partial y}\right)_{y=h_{1}} \\
& =(-m-2 a \pi \operatorname{Cos}[2 \pi x+\phi]) \\
& \left(\frac{-y N_{t}}{\left.(-2-2 m(t+x)-b \operatorname{Sin}[2 \pi x]-a \operatorname{Sin}[2 \pi x+\phi])^{2}\right)}\right) \\
& -(-2(1+m(t+x)+b \operatorname{Sin}[2 \pi x]) \\
& +2(-1-m(t+x)-a \operatorname{Sin}[2 \pi x+\phi]) \\
& -(1+m(t+x)+b \operatorname{Sin}[2 \pi x]) N_{t} \\
& -\frac{\left.(-1-m(t+x)-a \operatorname{Sin}[2 \pi x+\phi]) N_{t}\right)}{\left.\left(2(-2-2 m(t+x)-b \operatorname{Sin}[2 \pi x]-a \operatorname{Sin}[2 \pi x+\phi])^{2}\right)\right)^{\circ}}
\end{aligned}
$$

The non-dimensional average rise in pressure $\Delta p$ per wavelength at this order is given as follows

$$
\Delta \boldsymbol{p}_{1}=\int_{0}^{1}\left(\frac{\partial p_{1}}{\partial x}\right)_{y=0} d x .
$$

Where

$$
\boldsymbol{N}=\sqrt{M^{2}+\frac{1}{k}},
$$

$\mathbf{h} 1=-1-m(t+x)-a \operatorname{Sin}[2 \pi x+\phi]$,

$\mathbf{h} 2=1+m(t+x)+b \sin [2 \pi x]$,

$\boldsymbol{F}_{\mathbf{0}}=\boldsymbol{Q}+a \operatorname{Sin}[2 \pi x+\phi]+b \operatorname{Sin}[2 \pi x], \boldsymbol{F}_{\mathbf{1}}=0$

\section{Graphical Results and Discussion}

The graphical description of various parameters on flow of pseudoplasticnanofluids in the tapered asymmetric channel in this section. Particularly the results of pressure gradient distribution, average pressure rise, axial velocity, temperature distribution, nanoparticle distribution, heat transfer coefficient and stream function are recorded in terms of plots and then discussed physically. To study the behavior of solutions, numerical calculations for several values of amplitudes of lower and upper walls $(a \& b)$, has been evaluated numerically using MATHEMATICA and the results are presented graphically.

\subsection{The Pressure gradient distribution}

In this section, Figure (2)-(5) shows the pressure gradient $\frac{d p}{d x}$ for different value of thermal conductivity of fluid $\mathrm{k}$, the Hartman number $\mathbf{M}$, the phase difference $\phi$, non-uniform parameter of the channel $m$ and the values of $\mathrm{y}$ and $\mathrm{t}$ are fixed at $y=0.2$ and $t=0.1$. In all cases a relationship is observed between axial pressure gradient and volume flow rate, the Figure (2), We observed from the graph that the pressure gradient $\frac{d p}{d x}$ decreases at the core part of the channel. However opposite behavior is observed near channel boundaries with an increase in $\phi$.The Figure (3) and (4) shows that larger $Q$ and $\mathrm{M}$ results in the decrease of $\frac{d p}{d x}$. From Figure (5), we can conclude that the pressure gradient $\frac{d p}{d x}$ increases at the centre of the channel. However opposite behavior is observed near channel boundaries with an increase in $\xi$.

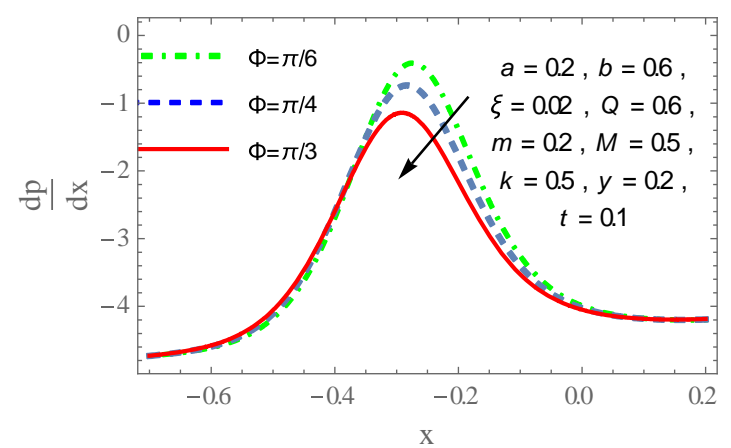

Figure 2: Variation of pressure gradient $\frac{d p}{d x}$ with different values of $\phi$

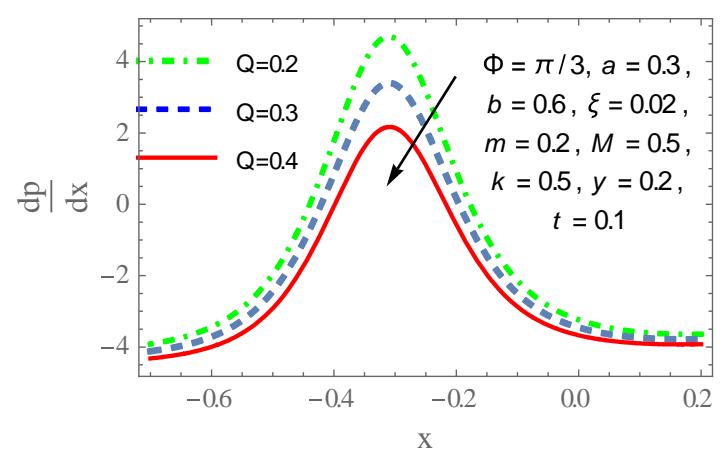

Figure 3: Variation of pressure gradient $\frac{d p}{d x}$ with different values of $Q$ 


\section{International Journal of Science and Research (IJSR) \\ ISSN (Online): 2319-7064}

Index Copernicus Value (2016): 79.57 | Impact Factor (2015): 6.391

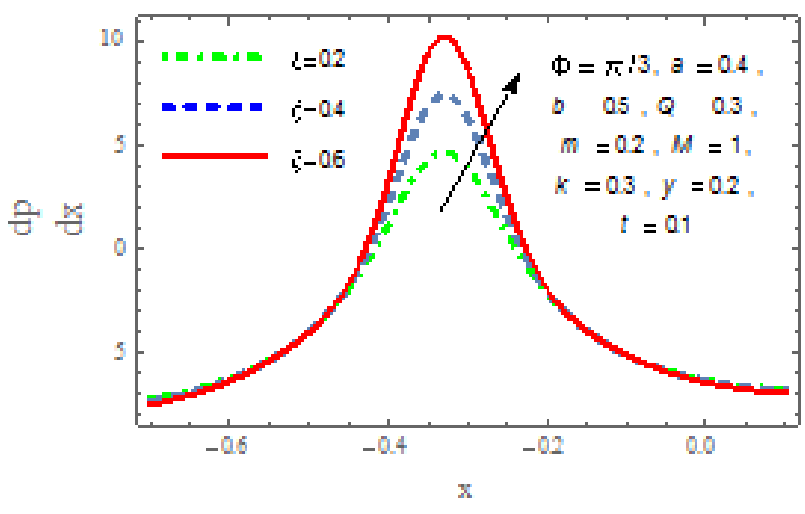

Figure 4: Variation of pressure gradient $\frac{d p}{d x}$ with different values of $M$

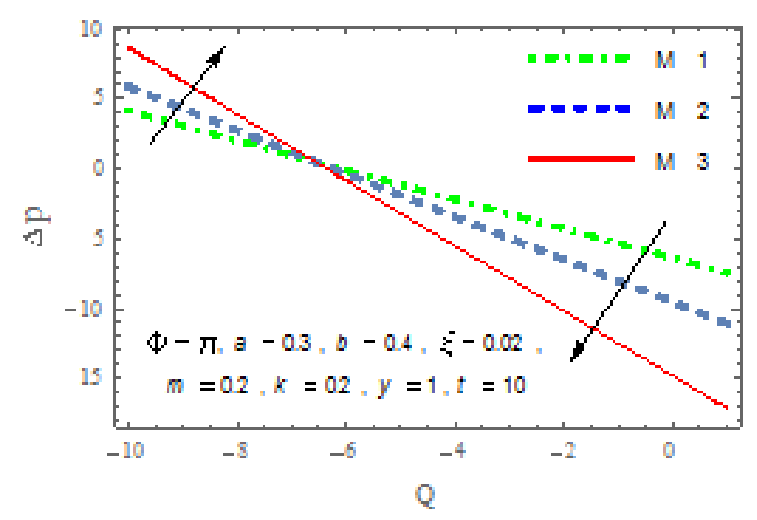

Figure 5: Variation of pressure gradient $\frac{d p}{d x}$ with different values of $\xi$

\subsection{Pumping Characteristics}

Figure (6)-(11) shows the variation of average pressure rise $\Delta p$ against time mean flow rate $Q$. The whole region is considered into five parts (I) $(\Delta p=0, Q>0)$ corresponds to the free pumpng region. (II) peristaltic pumping region where $(\Delta p>0, Q>0)$. (III) augmented pumping region where $(\Delta p<0, Q>0)$. (IV) when $(\Delta p>0, Q<0)$, then it is a backward pumping region. (V) There is a co-pumping region where $(\Delta p<0, Q<0)$. It is seen in Figure (6) it is noticed that by increase $\mathrm{k}, \Delta p$ decreases in the backward pumping regin till it reaches a critical value $Q=-6.4$ in the peristaltic pumping region after which $\Delta p$ increasing with increases k. Figure (7) Graph shows that the pumping rate an increase in the co-pumping region $(\Delta p<0, Q>0)$ with an increase in $a$. Figure (8) shows that an effect of $M$ on $\Delta p$. It is observed that free pumping regin $\Delta p=0$. Figure (9) shows the impact of $b$ on average pressure rise $\Delta p$. We observed from the graph that in co-pumping region $(\Delta p<0, Q>0)$, the pumping rate increase for larger $b$. Figure (10) also $(\Delta p<$ 0 , Q >augmented pumping region with increases $\phi$.Figure (11) shows that an effect of $m$ on $\Delta p$. It is observed that in backward pumping region or retrograde $(\Delta p<0, Q<$ $0.4)$ and the pumping rate enhances $(\Delta p<0, Q>0.4)$ with increase in $m$.

Currents are induced in the tissue or medium by the moving ions. This interaction serves as a basis of magnetically induced blood flow.

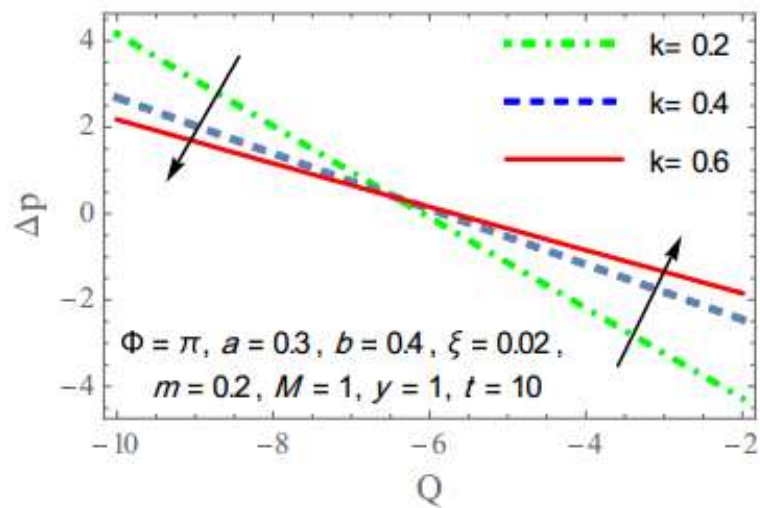

Figure 6: Pressure rise $\Delta p$ versus time-averaged flow rate $Q$ with different values of $k$

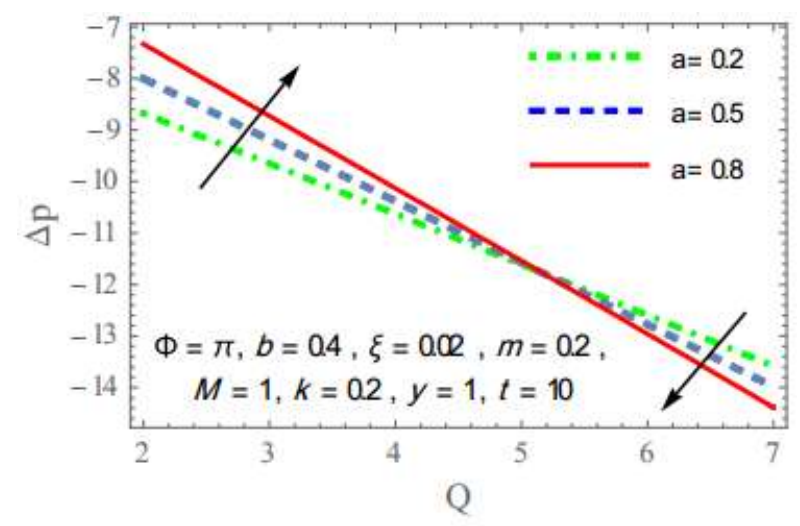

Figure 7: Pressure rise $\Delta p$ versus time-averaged flow rate $Q$ with different values of $a$

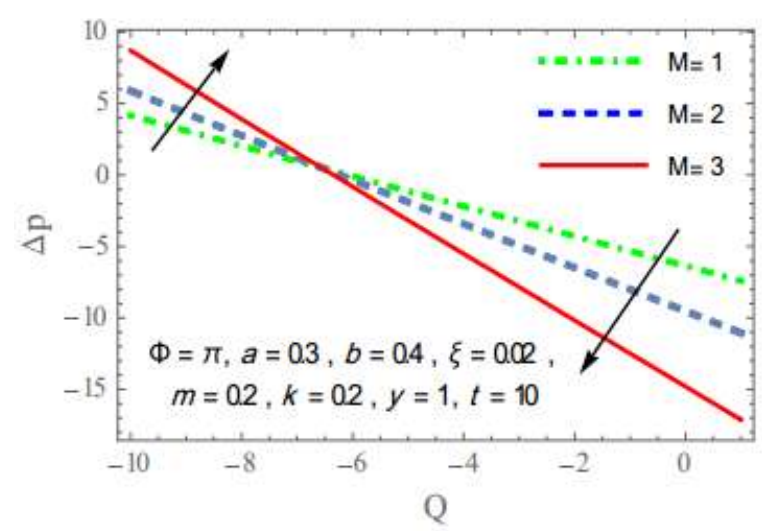

Figure 8: Pressure rise $\Delta p$ versus time-averaged flow rate $Q$ with different values of $M$

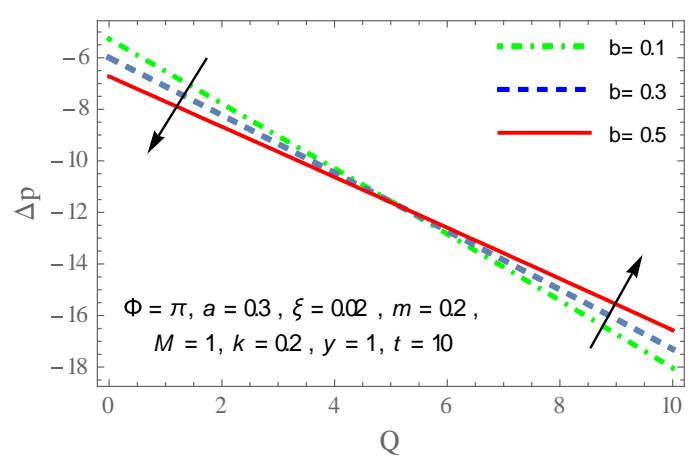

Figure 9: Pressure rise $\Delta p$ versus time-averaged flow rate $Q$ with different values of $b$ 


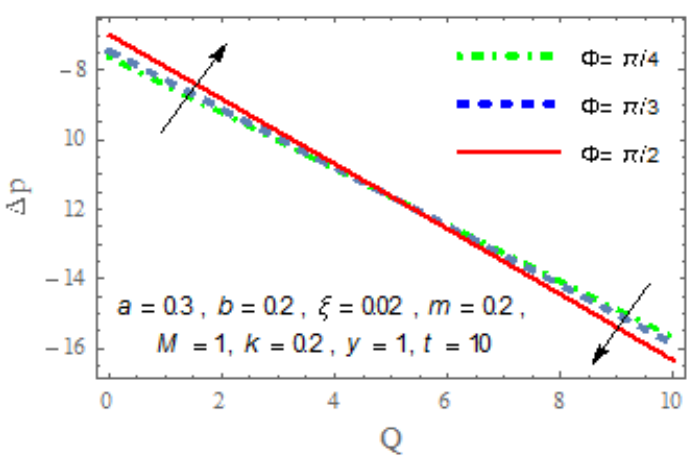

Figure 10: Pressure rise $\Delta p$ versus time-averaged flow rate $Q$ with different values of $\phi$

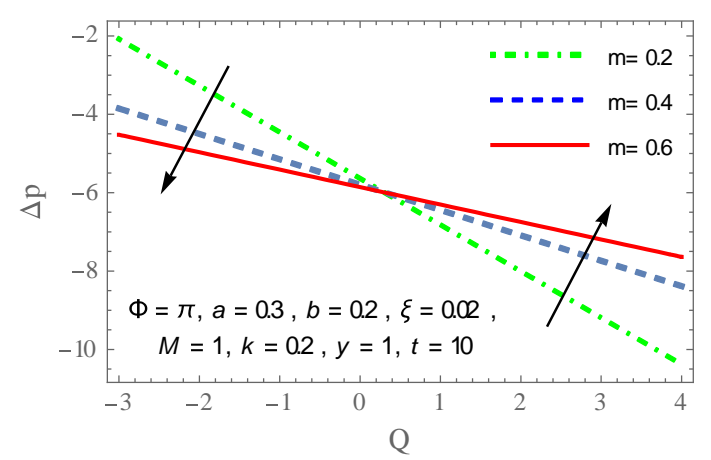

Figure 11: Pressure rise $\Delta p$ versus time-averaged flow rate $Q$ with different values of $m$

\subsection{Velocity Profile}

To see the effects of non-uniform parameter $m$, Hartmann number $M$, the amplitudes of waves along the lower and upper walls (a \& b), phase angle $\phi$, pseudoplastic fluid parameter $\xi$ and mean flow rate $Q$ on the velocity profile $\mathrm{u}$, the graphs are sketched in Figure (12)-(18) at the fixed values of $x=0.3$ and $t=2$. The behavior of velocity profile is parabolic as seen through figures. The velocity profile for the non-uniform parameter $m$ and Hartmann number $M$ are plotted in Figure (12) and(13). We observed from the graphs that the axial velocity $u$ increases with an increase in $m$ and $M$ at the core part of the channel. However opposite behavior is observed near channel boundaries. Physically speaking, the result of increasing magnetic field strength is to dampen the velocity. From Figure (14), it can be seen that impact $b$ on $u$ where decreases the axial velocity $u$ when an increase in b. But from Figure (15), illustrate an increase in a increases the axial velocity. Also the velocity profile for the phase angle $\phi$ is shown in Figure (16). It has been observed that an increase in $\phi$ values causes as an increase in the velocity profile $u$. It is examined in Figure (17) that by increasing $\xi$, the velocity of fluid $u$ is decreases. We observed from Figure (18) the velocity profile for the parameter $Q$ which depicts that the axial velocity $u$ decreases with an increase in $Q$ at the core part of the channel while we note that an increase in $Q$ causes as decrease in the magnitude of $u$ at the boundaries.

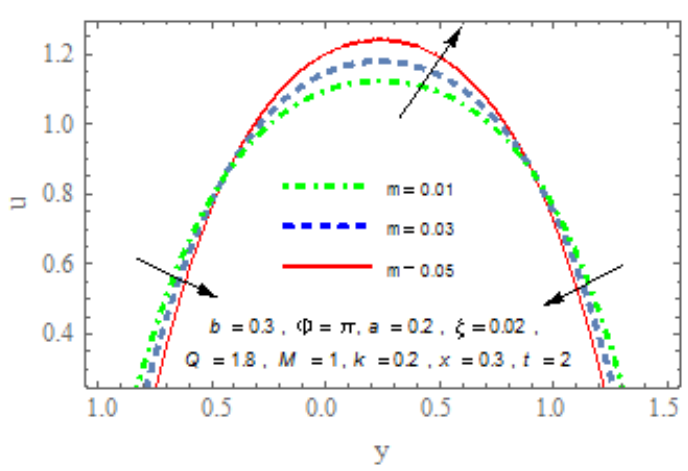

Figure 12: Axial velocity $u$ versus y- direction for different valuesm

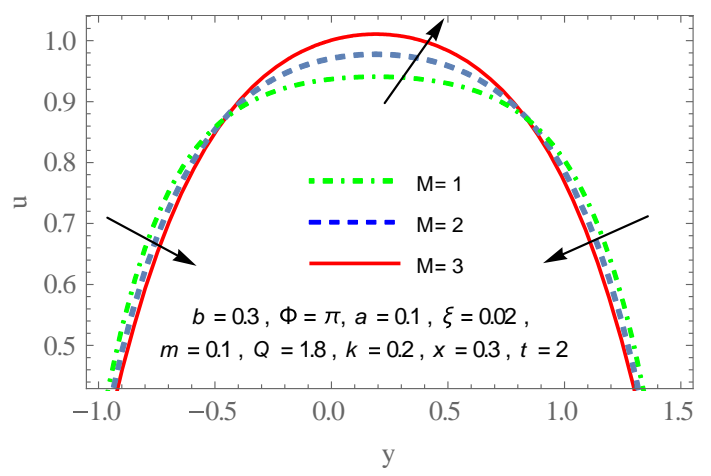

Figure 13: Axial velocity $u$ versus y- direction for different values $M$

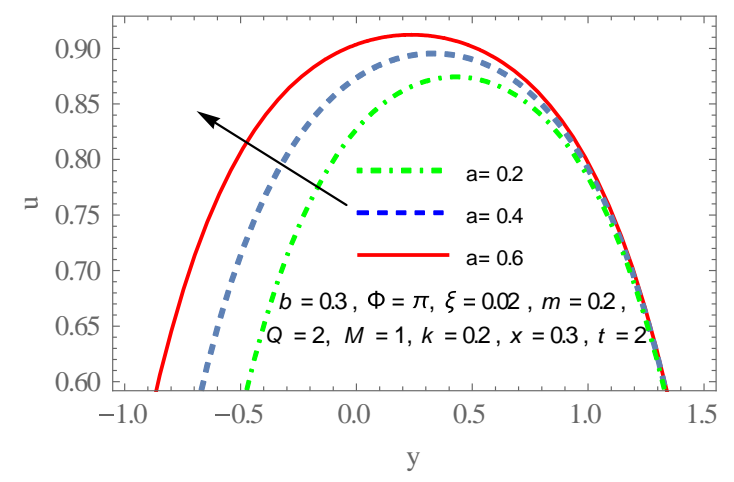

Figure 14: Axial velocity $u$ versus y-direction for different values $b$

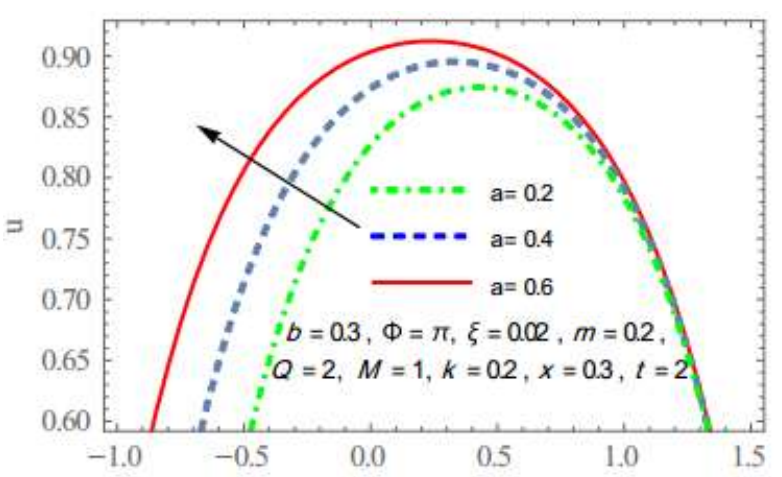

Figure 15: Axial velocity $u$ versus y- direction for different valuesa 


\section{International Journal of Science and Research (IJSR) \\ ISSN (Online): 2319-7064}

Index Copernicus Value (2016): 79.57 | Impact Factor (2015): 6.391

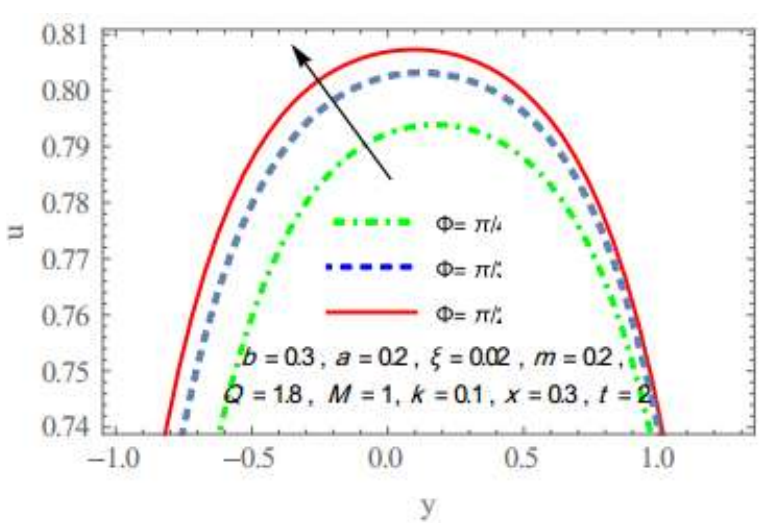

Figure 16: Axial velocity $u$ versus y- direction for different values $\phi$

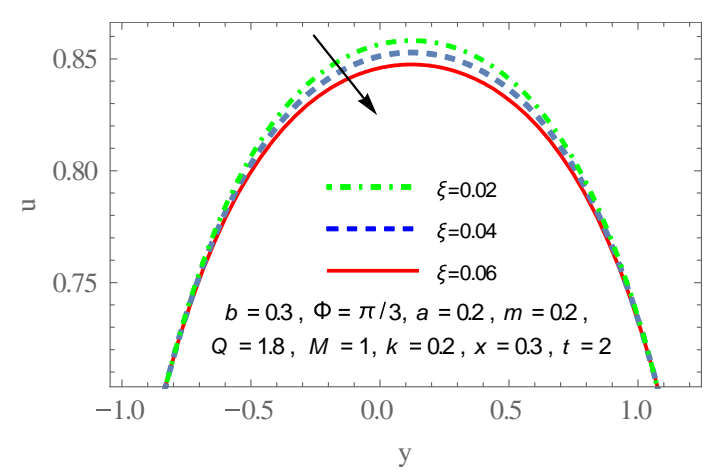

Figure 17: Axial velocity $u$ versus y- direction for different values $\xi$

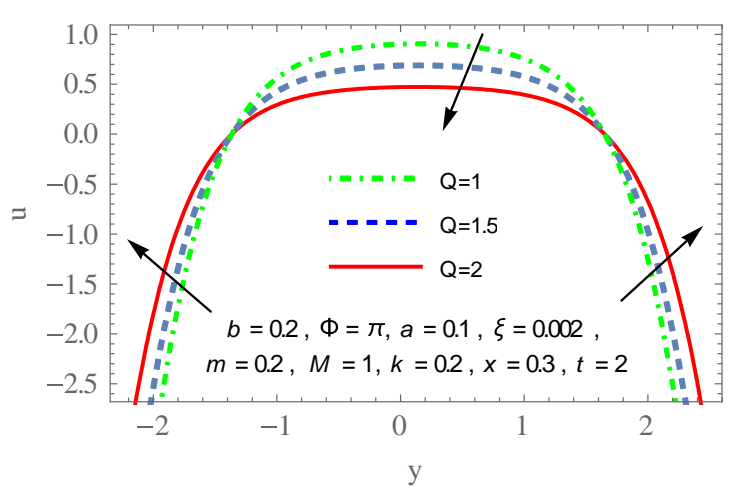

Figure 18: Axial velocity $u$ versus y-direction for different values $Q$

\subsection{Temperature distribution}

The influence of temperature of the fluid under the effect of peristalsis is exhibits in Figure (19)-(23) for the fixed values of $x=0.1$ and $t=0.2$. The impression of amplitude of upper wall $b$, on the temperature are shown in Figure (19). We note that the magnitude of temperature decreases with increasing $b$ in the vicinity of wall. Figure (20) it is seen that the temperature distribution enhances with an increase in $a$. The effects of increasing non-uniform parameter $m$ on the temperature are plotted in Figure (21). We observed that the temperature enhances with increase of $\mathrm{m}$ at the lower portion of the channel but effect is adverse at the upper part of the channel. While, it can be noted that the temperature at the lower wall is minimum and it augmented slowly towards the upper wall. Figure (22) and (23) are plotted to see that the temperature profile enhances with an increase in Prandtl number $\operatorname{Pr}$ and the thermophoresis parameter $N_{t}$, respectively.

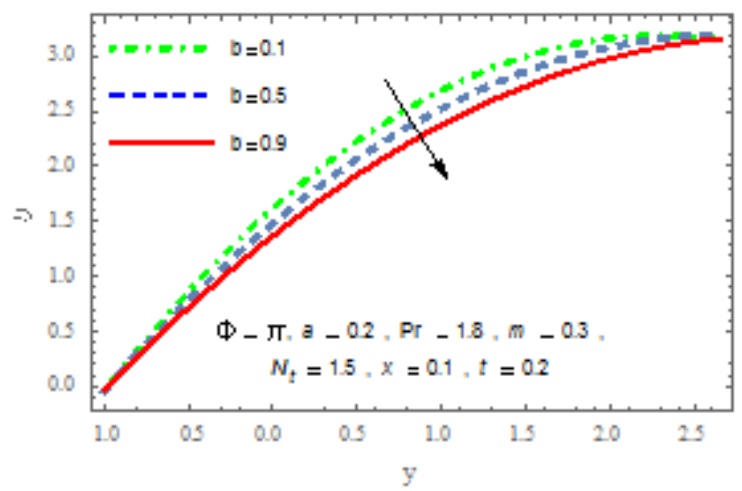

Figure 19: Temperature distribution $\theta$ for different values $b$

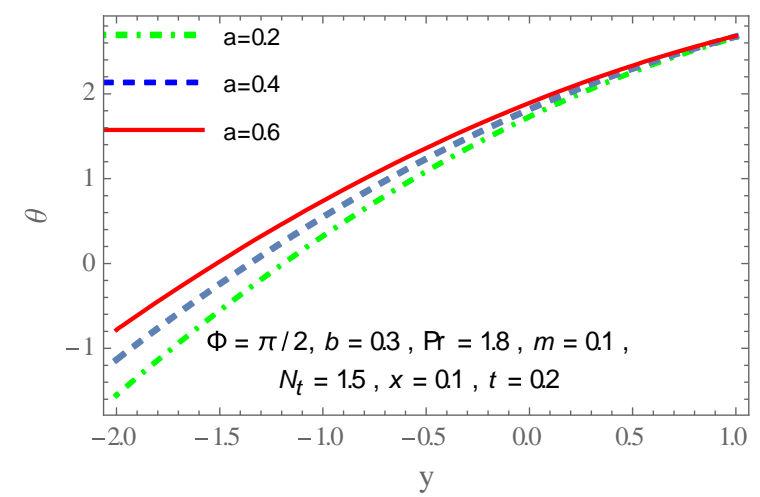

Figure 20: Temperature distribution $\theta$ for different values $a$

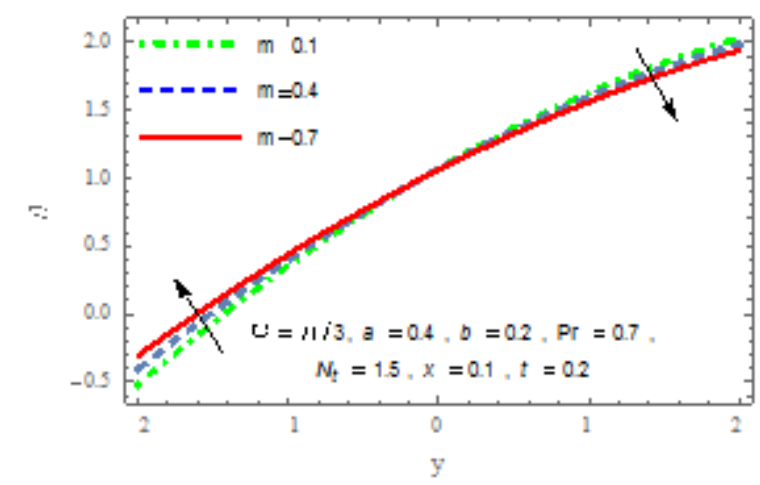

Figure 21: Temperature distribution $\theta$ for different values $m$

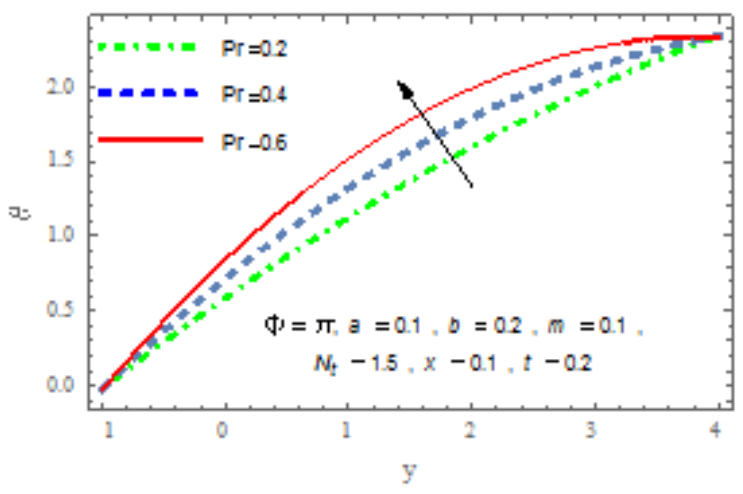

Figure 22: Temperature distribution $\theta$ for different values $P r$

\section{Volume 6 Issue 12, December 2017 www.ijsr.net}


International Journal of Science and Research (IJSR)

ISSN (Online): 2319-7064

Index Copernicus Value (2016): 79.57 | Impact Factor (2015): 6.391

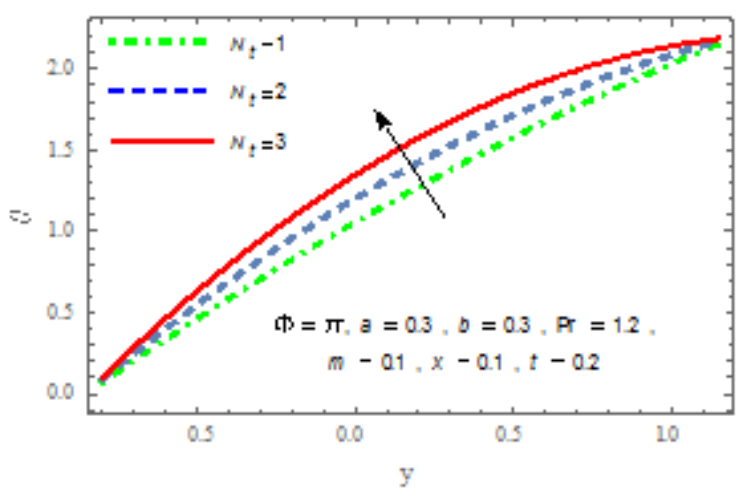

Figure 23: Temperature distribution $\theta$ for different values $N_{t}$

\subsection{Heat transfer coefficient}

Figure (24)-(26) are drawn to examine the effect of Prandtl number Pr the thermophoresis parameter and the amplitudes of wave along lower wall on heat transfer coefficient $Z$. It is seen that due to shrinkage and expansion of peristaltic channel walls the behavior of heat transfer coefficient $Z$ is wobbling. Figure (24) shows that absolute value of $Z$ decreases with an increase in Pr for the interval $(-0.45<$ $x<0.125$ and $Z$ increases with an increase in $\operatorname{Pr}$ for the interval $(0.125<x<0.5)$. Figure (25) exhibits absolute value of $Z$ increase with an increase in $N_{t}$ for the period $(-0.7<x<-0.22)$ and $Z$ decreases with an increase in $N_{t}$ for the interval $(-0.22<x<0.2)$. Finally the Figure (26) shows that the decreasing response of absolute heat transfer coefficient $Z$ with an increase ina for $(-0.5<x<0)$ and an increase with an increase in $a$ for $(0<x<0.5)$.

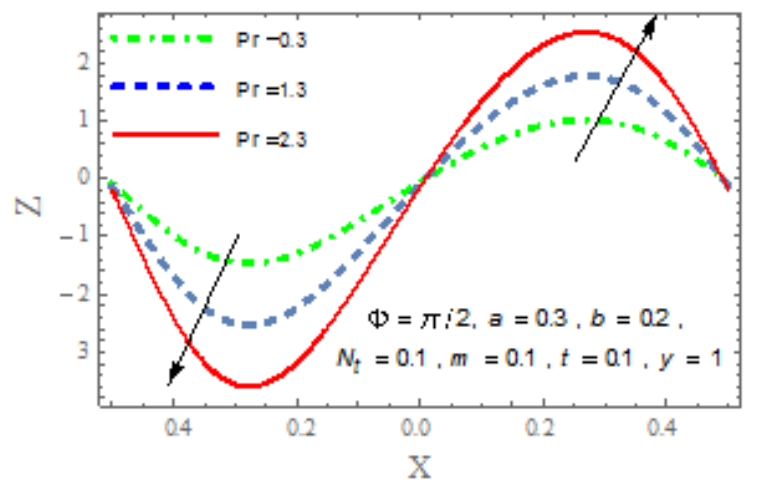

Figure 24: Heat transfer coefficient $\mathrm{Z}(\mathrm{x})$ for different values of $\mathrm{Pr}$

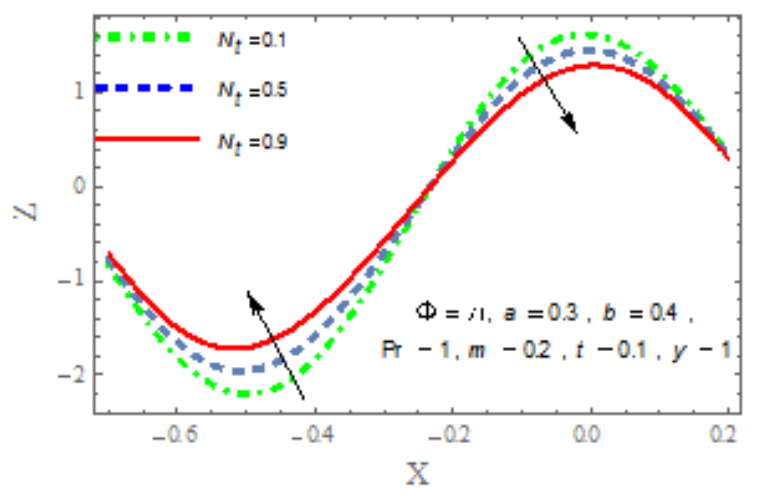

Figure 25: Heat transfer coefficient $\mathrm{Z}(\mathrm{x})$ for different values of $N_{t}$

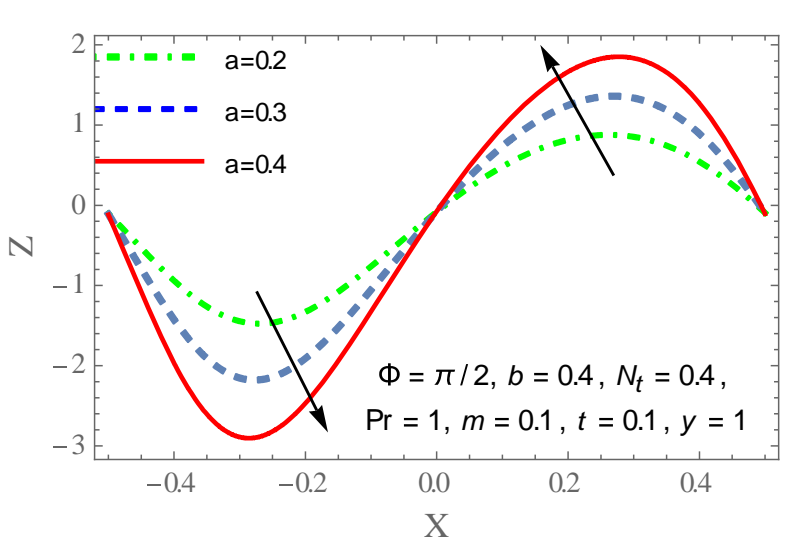

Figure 26: Heat transfer coefficient $\mathrm{Z}(\mathrm{x})$ for different values of $a$

\subsection{Trapping}

In this chapter, we study trapping for various values of lower and upper walls where the formation of an internally circulating bolus of fluid by closed streamlines is called trapping, the phenomenon of trapping is another interesting area in peristaltic transport. In a reference wave frame, this trapped bolus is boost ahead along with the peristaltic wave. It is attention to notice that bolus also illustrate in the fixed frame which may probably be due to the impression of nonzero time-average of the flow over one period of the wave. The trapping for different values of $m, a, Q$ and $\xi$ are shown in Figure (27)-(30) at fixed values of $t=0.1$.In this figures, we note that the significant variation and the strong impact of occurs bolus near on walls with high deformations. Figure (27) shows that the effect of uniform $(m=0.0)$ and non-uniform $(m=0.05)$ on the trapping. It is examined that the size of trapped bolus decreases with an increase in $m$. From Figure (28) we observed for the different values a on the streamlines. Exhibits the volume of the circulating bolus enhances with increasing $a$. The effect of the mean flow rate $Q$ on the streamlines is observed in Figure (29). We found that with the ascending values of $Q$, the trapping bolus decreases in size. Impact of pseudoplastic fluid parameter $\xi$ on the streamlines is examined in Figure (30).it is observed that for increasing value of $\xi$, the size of trapping bolus is slowly increasing.

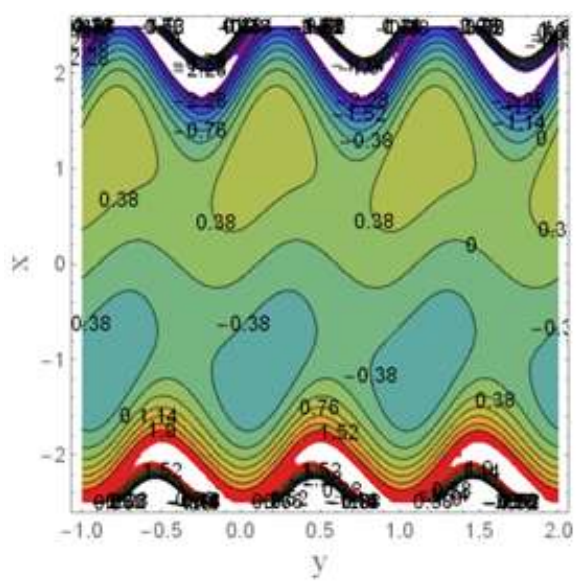

(a)

Volume 6 Issue 12, December 2017 www.ijsr.net 
International Journal of Science and Research (IJSR)

ISSN (Online): 2319-7064

Index Copernicus Value (2016): 79.57 | Impact Factor (2015): 6.391

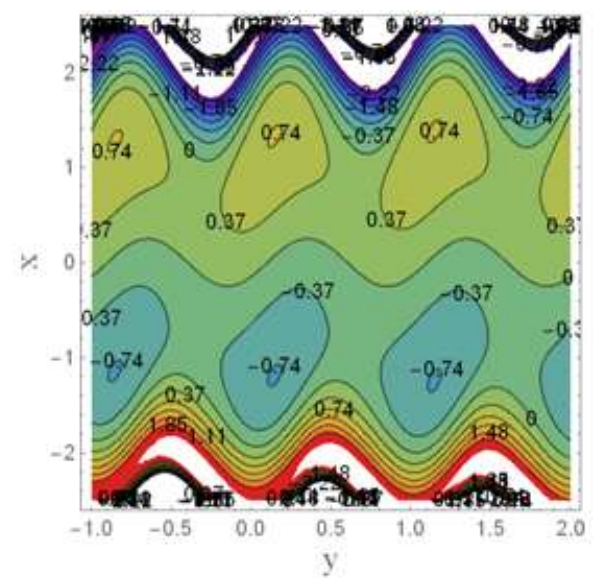

(b)

Figure 27: Streamlines for $b=0.4, \phi=\frac{\pi}{2}, a=0.4, M=$ $0.5, \xi=0.002, Q=1, k=0.2, t=0.1$ and for different $m:(\mathbf{a}) m=0.0,(\mathbf{b}) m=0.05$

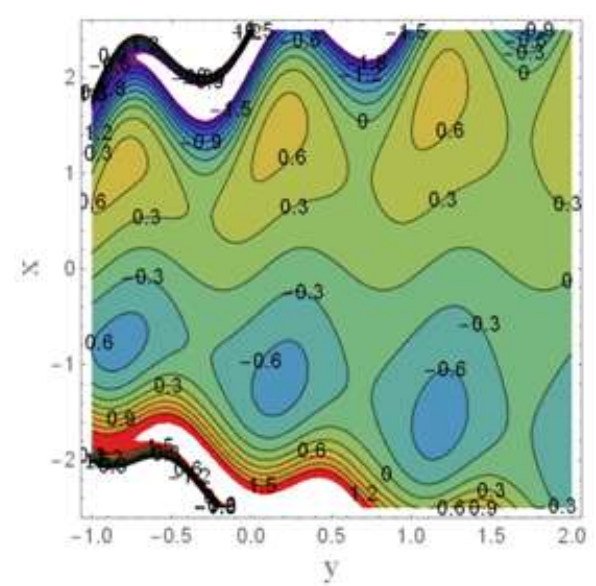

(a)

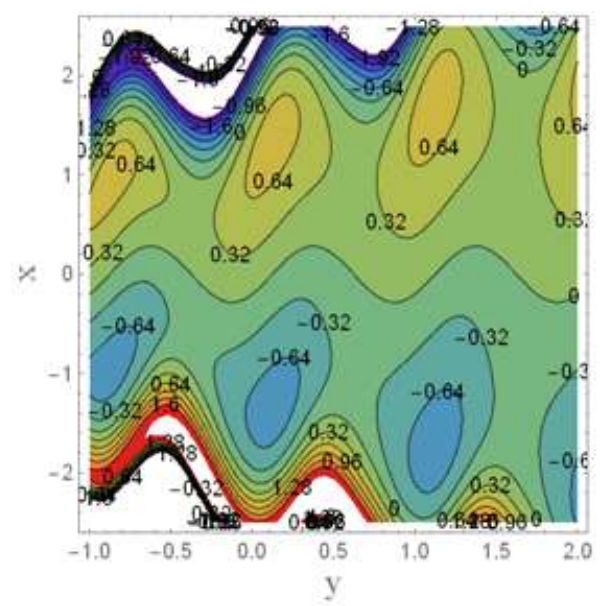

(b)

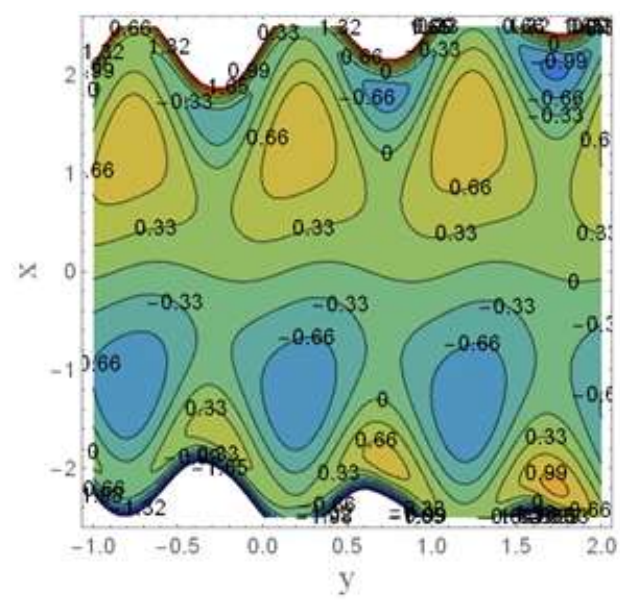

Figure 28: Streamlines for $b=0.4, \phi=\pi / 2, M=0.5, \xi=$ $0.002, Q=1.5, m=0.4, k=0.2, \mathrm{t}=0.1$ and for different $\mathrm{a}:(\mathbf{a}) \mathrm{a}=0.2,(\mathbf{b}) \mathrm{a}=0.4$

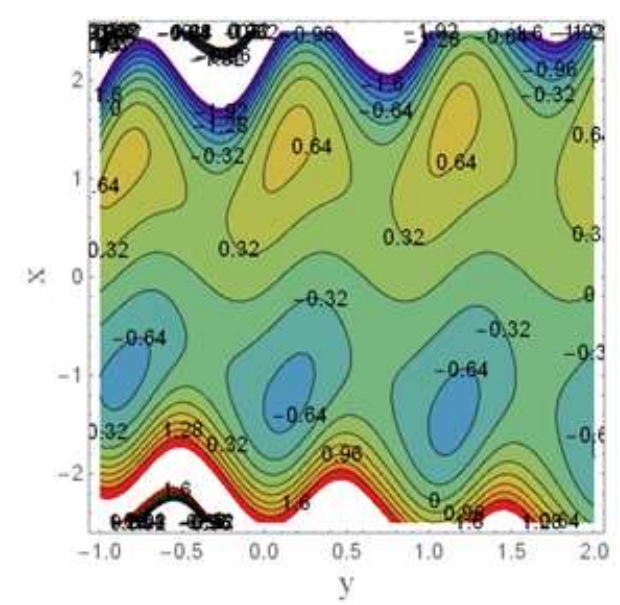

(a)

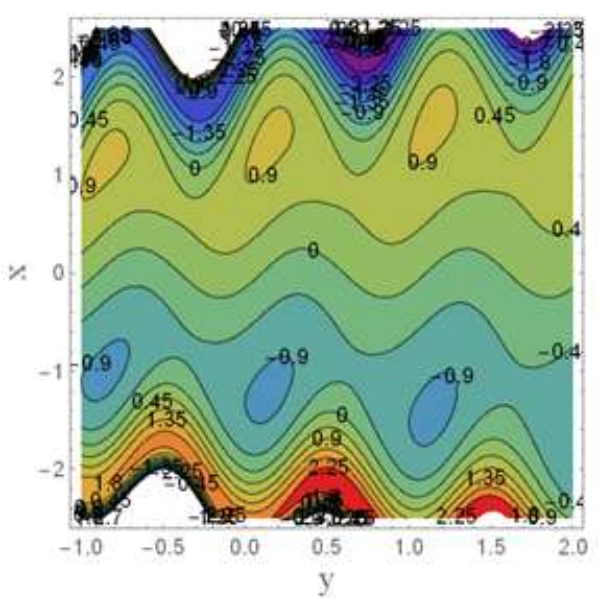

(b)

Figure 29: Streamlines for $b=0.4, \phi=\pi / 2, a=0.3, M=$ $0.5, \xi=0.003, \mathrm{~m}=0.2, \mathrm{t}=0.1, \mathrm{k}=0.3$ and for different $\Theta:(\mathbf{a}) \mathrm{Q}=1,(\mathbf{b}) \mathrm{Q}=1.5$ (a)

Volume 6 Issue 12, December 2017 


\section{International Journal of Science and Research (IJSR) ISSN (Online): 2319-7064}

Index Copernicus Value (2016): 79.57 | Impact Factor (2015): 6.391

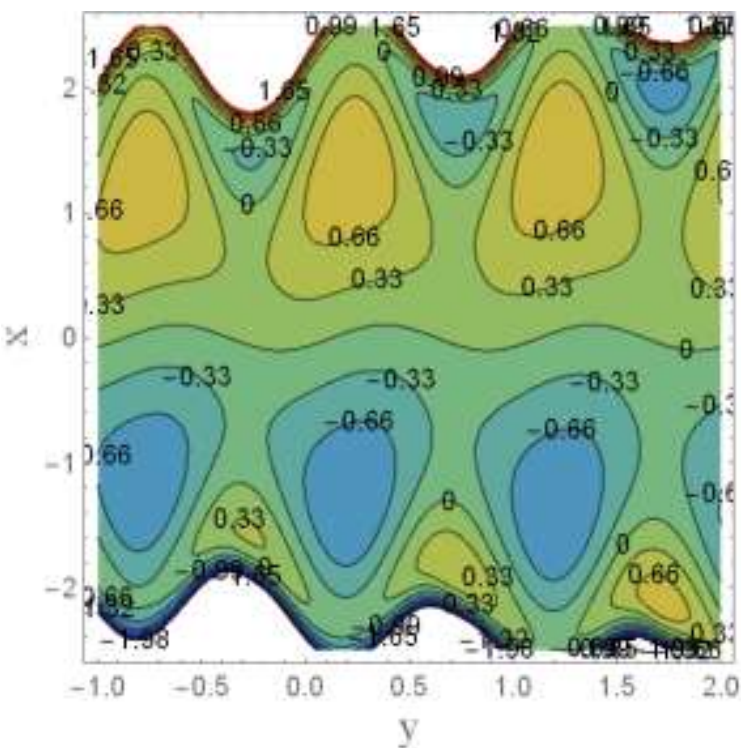

(b)

Figure 30: Streamlines for $b=0.4, \phi=\pi / 6, a=0.3, M=$ $0.5, \mathrm{Q}=1.2, \mathrm{~m}=0.1, \mathrm{t}=0.1, \mathrm{k}=0.4$ and for different

$$
\xi:(\mathbf{a}) \xi=0.02,(\mathbf{b}) \xi=0.025
$$

\section{Conclusions}

In this paper we study and examined a mathematical model of the peristaltic transport of a pseudoplasticnanofluid in the tapered asymmetric channel with the effect of magnetic field and porous medium. The channel asymmetry is a peristaltic wave train on the non-uniform walls to have different amplitudes and phase. A long-wavelength and low-Reynolds number approximations are adopted. A regular perturbation method is appoint to obtain the expression for the pressure gradient distribution, the pressure rise, the stream function, a velocity profile and the temperature distribution of the fluid with peristaltic flow is discussed the main results can be summarized as follows:

- The pressure gradient decreases with increase in $\phi, Q$ and M while it increase for decreasing $\xi$

- It has been found that the average rise in pressure $\Delta p$ enhances with the increase of $a, \phi, m$ and $\mathrm{M}$ while it decreases by increasing $k$ and $\mathrm{b}$.

- The velocity profile increases with increase $a$ and $\phi$ while it decreases by increasing $b$ and $\xi$.

- The axial velocity decreases with an increase in $Q$ at the core part of the channel. However opposite behavior on a near channel boundaries.

- An increase in $\mathrm{m}$ and Hartmann number $M$ results in increases of fluid velocity at the core part of the channel whereas it is different towards walls.

- The temperature distribution is decreases with increase $b$ and $\phi$ while it increases by increasing $a$ and $\xi$.

- A temperature of fluid is enhances near the lower wall of channel while it decreases in the upper wall when nonuniform parameter $m$ is increased.

- Temperature profile is an increasing function with increase in $a, N_{t}$ and $P_{r}$.

- Heat transfer coefficient is a decreasing function with an increase in $a$ and $P_{r}$ while it enhances by increasing $N_{t}$.
- The trapping bolus decreases with increasing the mean flow rate $Q$ and non-uniform parameter $m$ while it has a reverse behavior with increasing of a and pseudoplastic fluid parameter $\xi$.

\section{References}

[1] A. A. Shaaban, Mohamed Y. Abou-Zeid, Effects of heat and mass transfer on MHD peristaltic flow of a nonNewtonian fluid through a porous medium between two coaxial cylinders, Math. Prob. Eng. (2013).

[2] A. H Shapiro, M. Y. Jafferin, S. 1. Weinberg, Peristaltic pumping with long wavelength at low Reynolds number, J. Fluid Mech. 37 (1969)799-825.

[3] C. Barton, S Raynor, Peristaltic flow in tubes, Bull. Math. Biophys. 30 (1968) 663-680.

[4] Ellahi R., Riaz A., Nadeem S., (2013) "Threedimensional peristaltic flow of Williamson fluid in a rectangular duct”, Indian J. Phys. 87, 1275-1281.

[5] Eytan O., Jaffa A. J., Har-Toor J., Dalach E., Elad D., (1999) "Dynamics of the intrauterine fluid-wall interface", Ann. Biomed. Eng. 27, 372-379.

[6] F. M. Abbasi, T. Hayat, A. Alsaedi, B. Ahmed, Soret and Dufour effects on peristaltic transport of MHD fluid with variable viscosity, Appl. Math. Inf. Sci. 8 (2014) 211219.

[7] G. Radhakrishnamacharya, Ch. Srinivasulu, Influence of wall properties on peristaltic transport with heat transfer, C. R. Mec. 335 (2007) 369-373.

[8] H. Yasmin, T. Hayat, N. Alotaib, H. Gao, Convective heat and mass transfer analysis on peristaltic flow of Williamson fluid with Hall effects and Jouleheating, Int. J. Biomath.

(2014), http://dx.doi.org/10.1142/S1793524514500582.

[9] K. Nowar, Peristaltic flow of a nanofluid under the effect of Hall current and porous medium, Math. Prob. Eng (2014).

[10] M. Kothandapani, J. Prakash, Convective boundary conditions effect on peristaltic flow ofa MHD Jeffrey nanofluid, Appl. Nanosci. (2015), http://dx.doi.org/10.1007/ s13204-015-0431-9.

[11] M. Mustafa, S. Abbasbandy, S.Hina, T.Hayat, Numerical investigation on mixed convective peristaltic flow of fourth grade fluid with Dufour and Soret effect, J. TaiwanInst. Chem. Eng. 45 (2014) 308-316.

[12] M. Kothandapani, J. Prakash, Effects of thermal radiation parameter and magnetic field on the peristaltic motion of Williamson nanofluids in a tapered asymmetric channel, Int. J. Heat Mass Transf. 81 (2015) 234-245.

[13]R. Ellahi, M. M. Bhatti, K. Vafai, Effects of heat and mass transfer on peristaltic flow in a non-uniform rectangular duct, Int. J. Heat Mass Transf. 71 (2014) 706-719.

[14] T. Hayat, A. Tanveer, F. Alsaadi, G. Mousa, Impact of radial magnetic field on peristalsis incurved channel with convective boundary conditions, J. Magn. Magn. Mater.403 (2016) 47-59.

[15] T.Hayat, F. M. Abbasi, Maryem Al-Yami, ShathaMonaquel, Slip and Joule heating effects in mixed convection peristaltic transport of nanofluid with Soret and Dufoureffects, J. Mol. Liq. 194 (2014) 93-99. 


\section{International Journal of Science and Research (IJSR) \\ ISSN (Online): 2319-7064}

Index Copernicus Value (2016): 79.57 | Impact Factor (2015): 6.391

[16] T. Hayat, R. Iqbal, A.Tanveer, A. Alsaedi, Influence of convective conditions in radiativeperistaltic flow of pseudoplasticnanofluid in a tapered asymmetric channel, J.Magn.Magn. Mater. 408 (2016) 168-176.

[17] T.Hayat, RijaIqba, A.Tanveer, A. Alsaedi, Soret and Dufour effects in MHD peristalsis of pseudoplasticnanofluid with chemical reaction, J. Mol. Liq. August 2016, pages 693-706

[18] T. W. Latham, Fluid motion in a peristaltic pump, MS Thesis, MiT, USA, 1966.

[19] Y. C Fung, C. S. Yih, Peristaltic transport, ASME J, APPl. Mech. 35 (1968) 669-675.

\section{Author Profile}

Ahmad M. Abdulhadi I received the B.S and MS.c degree from Baghdad University -college of science-department of mathematics 1988. The Ph.D from Pune University, India in 2000. I have degree of professor in mathematics since 2012.Now, working in college of science-department of mathematics.

Mohammed R Salman: I received the B.S degree from Al- Mustansiriyah university-college of science-department of mathematics 1989 and MS. degree from Al-Kufa university-college of education for women-department of mathematics 2014.

Volume 6 Issue 12, December 2017 